\title{
Effects of PEG anchors in PEGylated siRNA lipoplexes on in vitro gene-silencing effects and siRNA biodistribution in mice
}

\author{
YOSHIYUKI HATTORI $^{1,2}$, KYOKO TAMAKI $^{2}$, SHO SAKASAI $^{1,2}$, KEI-ICHI OZAKI $^{3}$ and HIRAKU ONISHI ${ }^{2}$ \\ Departments of ${ }^{1}$ Molecular Pharmaceutics and ${ }^{2}$ Drug Delivery Research, Hoshi University, \\ Tokyo 142-8501; ${ }^{3}$ Department of Molecular Pathology, Faculty of Pharmaceutical Sciences, \\ Doshisha Women's College of Liberal Arts, Kyotanabe, Kyoto 610-0395, Japan
}

Received April 7, 2020; Accepted August 3, 2020

DOI: $10.3892 / \mathrm{mmr} .2020 .11525$

\begin{abstract}
Polyethylene glycol (PEG)-modifications (PEGylations) of cationic liposome/small interfering RNA complexes (siRNA lipoplexes) can enhance their systemic stability. The present study determined the effects of PEG anchors in PEGylated siRNA lipoplexes on in vitro gene-silencing effects and siRNA biodistribution after intravenous injection. Three types of dialkyl or trialkyl cationic lipids were used in the current study for the preparation of cationic liposomes. Additionally, various PEGylated siRNA lipoplexes that contained PEG-1,2-distearoyl-snglycero-3-phosphoethanolamine (DSPE), PEG-1,2-di stearoyl-rac-glycero-3-methylpolyoxyethylene (DSG), PEG-cholesterol (PEG-Chol) and PEG-chondroitin sulfate conjugate (PEG-CS) were prepared. The results revealed that PEGylation of siRNA lipoplexes with PEG-DSPE strongly decreased gene-silencing effects in cells. In contrast, those with PEG-DSG, PEG-Chol and PEG-CS did not largely decrease gene-silencing effects. However, regardless of the PEG-derivative type, PEGylation of siRNA lipoplexes decreased their agglutination with erythrocytes. Furthermore, intravenous injection of PEGylated siRNA lipoplexes markedly decreased the accumulation of siRNA in the lungs, regardless of the type of PEG-derivative. However, non-PEGylated siRNA lipoplexes accumulated mainly in the lungs regardless of the siRNA lipoplex cationic lipid type. The results indicated that PEGylation of siRNA lipoplexes with PEG-DSG, PEG-Chol and PEG-CS may improve systemic stability without losing transfection activity by PEGylation.
\end{abstract}

Correspondence to: Professor Yoshiyuki Hattori, Department of Molecular Pharmaceutics, Hoshi University, 2-4-41 Ebara, Shinagawa-ku, Tokyo 142-8501, Japan

E-mail: yhattori@hoshi.ac.jp

Key words: cationic liposome, polyethylene glycol modification, small interfering RNA delivery, gene knockdown

\section{Introduction}

The introduction of synthetic small interfering RNAs (siRNAs) into cells can elicit RNA interference (RNAi), thereby inhibiting the expression of a targeted mRNA in the cells (1). Therefore, siRNA therapeutics has great potential to become the next generation of medicines. The effectiveness of siRNA therapeutics relies on an siRNA delivery system that can protect siRNAs from serum nucleases and deliver siRNAs efficiently into cells in a target tissue (2). In particular, cationic liposomes have been widely investigated for in vitro and in vivo siRNA delivery $(3,4)$. For systemic siRNA delivery with cationic liposomes, siRNA/cationic liposome complexes (siRNA lipoplexes) must be stabilized in the blood by avoiding their interaction with blood components such as erythrocytes (5). Therefore, polyethylene glycol (PEG)-modified cationic liposomes are often used for in vivo siRNA transfection, and a PEG-lipid derivative is incorporated into the liposomal membrane via a lipid-anchor during the preparation of liposomes. PEG modification (PEGylation) on the surface of siRNA lipoplexes can protect siRNA lipoplexes from interaction with blood components and macrophage capture, and consequently prolong retention in the blood circulation (6). Despite the advantages of PEGylation, the presence of PEG on the surface of siRNA lipoplexes severely decreases the gene-silencing efficiency of siRNA lipoplexes by preventing cellular uptake and endosomal escape processes, which is known as the PEG dilemma (6,7).

Generally, PEGylation of siRNA lipoplexes can reduce siRNA accumulation in the lungs by avoiding agglutination with blood components. However, PEGylation with 1-2 mol\% PEG-1,2-distearoyl-sn-glycero-3phosphoethanolamine (PEG-DSPE) strongly inhibited the gene-silencing effects by siRNA lipoplexes (8), indicating that PEGylation with PEG-DSPE abolished gene-silencing by siRNA lipoplexes with increasing content of PEG-DSPE in liposomal formulations. Therefore, for the development of siRNA lipoplexes without the loss of gene-silencing activity by PEGylation, an optimal amount of PEG-lipid must be included in the PEGylated liposomal formulation. Regarding PEG-lipid derivatives, 1,2-distearoyl-rac-glycero3-methylpolyoxyethylene (PEG-DSG), 1,2-dimyristoyl-racglycero-3-methylpolyoxyethylene (PEG-DMG), and 
poly(ethylene glycol) cholesteryl ether (PEG-Chol) have also been used for the PEGylation of cationic liposomes. Inclusion of PEG-lipid with longer saturated diacyl chains into siRNA lipoplexes increased the plasma concentration of lipoplexes after intravenous injection (9), indicated that the length and saturation of the acyl chain in PEG-lipid derivatives strongly affected the stability of PEGylated siRNA lipoplexes in the blood circulation after intravenous injection. PEGylated siRNA lipoplexes with PEG-DSPE showed excellent blood circulation compared with those incorporating PEG-DMG (10). In addition, PEG-DMG dissociated rapidly from PEGylated lipid nanoparticle-encapsulated siRNA, but PEG-DSG dissociated slowly (11). Furthermore, incorporation of PEG-Chol into cationic liposomes was stabilized in human urine and enhanced cellular uptake, whereas the incorporation of PEG-DSPE or PEG-DSG into cationic liposomes effectively prevented the formation of agglomeration but decreased cellular uptake (12). These findings suggested that anchors of PEG derivatives in PEGylated siRNA lipoplexes strongly affected cellular association and stability in the blood circulation. However, to the best of our knowledge, there are still few reports about the effects of PEG anchors in PEGylated siRNA lipoplexes on gene-silencing activity and siRNA biodistribution.

In this study, to examine the effects of PEG anchors in PEGylated siRNA lipoplexes on in vitro gene-silencing and siRNA biodistribution after intravenous injection, we used various kinds of PEG derivatives, and prepared PEGylated siRNA lipoplexes. Here, we found that PEGylation of siRNA lipoplexes with PEG-DSG, PEG-Chol, and PEG-chondroitin sulfate conjugate (PEG-CS) might improve the systemic stability without loss of transfection activity by PEGylation.

\section{Materials and methods}

Materials. 1,2-Dioleoyl-3-trimethylammonium-propane methyl sulfate salt (DOTAP) was obtained from Avanti Polar Lipids Inc. (Alabaster, AL, USA). Dimethyldioctadecylammonium bromide (DDAB, product name: DC-1-18) and 11-((1,3-bis(dodecanoyloxy)-2-((dodecanoyloxy) methyl)propan-2-yl)amino)- $N, N, N$-trimethyl-11-oxoundecan1-aminium bromide (product name: TC-1-12) were obtained from Sogo Pharmaceutical Co., Ltd. (Tokyo, Japan). $N$-(Methylpolyoxyethylene oxycarbonyl)-1,2-distearoyl$s n$-glycero-3-phosphoethanolamine, sodium salt (PEG mean molecular weight (MW): 2000, PEG-DSPE, SUNBRIGHT ${ }^{\circledR}$ DSPE-020CN), $N$-(Methylpolyoxyethylene oxycarbonyl)1,2-dipalmitoyl-sn-glycero-3-phosphoethanolamine, sodium salt (PEG mean MW: 2000, PEG-DPPE, SUNBRIGHT ${ }^{\circledR}$ PP-020CN), $N$-(Methylpolyoxyethylene oxycarbonyl)-1,2-dimy ristoyl-sn-glycero-3-phosphoethanolamine, sodium salt (PEG mean MW: 2000, PEG-DMPE, SUNBRIGHT ${ }^{\circledR}$ PM-020CN), 1,2-distearoyl-rac-glycero-3-methylpolyoxyethylene (PEG mean MW: 2000, PEG-DSG, SUNBRIGHT ${ }^{\circledR}$ GS-020), 1,2-dipalmitoyl-rac-glycero-3-methylpolyoxyethylene (PEG mean MW: 2000, PEG-DPG, SUNBRIGHT ${ }^{\circledR}$ GP-020), 1,2-dimyristoyl-rac-glycero-3-methylpolyoxyethylene (PEG mean MW: 2000, PEG-DMG, SUNBRIGHT ${ }^{\circledR}$ GM-020), poly(ethylene glycol) cholesteryl ether (PEG mean MW: 2000, PEG-Chol, SUNBRIGHT ${ }^{\circledR}$ CS-020), and 1,2-dioleoyl-sn-glycero-3-phosphoethanolamine (DOPE,
COATSOME ME-8181) were obtained from NOF Co., Ltd. All other chemicals were of the highest grade available.

Small interfering RNAs. siRNAs targeting nucleotides of firefly luciferase (Luc siRNA), non-silencing siRNA (control [Cont] siRNA) as a negative control for Luc siRNA, and cyanine 5.5 (Cy5.5)-labeled pGL3 luciferase siRNA (Cy5.5-siRNA) were synthesized by Sigma Genosys. The siRNA sequences of the Luc siRNA were: Sense strand: 5'-CCGUGGUGUUCGUGU CUAAGA-3', and antisense strand: 5'-UUAGACACGAAC ACCACGGUA-3 (13). The siRNA sequences of the Cont siRNA were: Sense strand: 5'-GUACCGCACGUCAUUCGU AUC-3', and antisense strand: 5'-UACGAAUGACGUGCG GUACGU-3' (14). The siRNA sequence of Cy5.5-siRNA was as reported previously (15).

Synthesis of PEG-CS. Methoxypolyethylene glycolchondroitin sulfate conjugate (PEG-CS) was produced by periodate oxidation of chondroitin sulfate $\mathrm{C}$ sodium salt (CS, MW 35,000; Wako Pure Chemical Industries, Ltd.) (16) and subsequent reductive amination with methoxypolyethylene glycol amine (mPEG-NH ${ }_{2}$; MW 2,000, SUNBRIGHT ${ }^{\circledR}$ MEPA-20H; NOF Co., Ltd.) (17) as described as follows. After CS (1 g) was dissolved in water $(20 \mathrm{ml}), \mathrm{NaIO}_{4}(0.3 \mathrm{~g})$ was added and stirred in the dark at room temperature for $4 \mathrm{~h}$. The mixture was chromatographed with a Sephadex G50 (GE Healthcare Bio-Science AB) column ( $3 \mathrm{~cm}$ in inner diameter $\mathrm{x} 20 \mathrm{~cm}$ in length) using $0.1 \mathrm{M} \mathrm{NaCl}$ aqueous solution. The high molecular weight fractions were put into a cellulose tube (MW cut-off 12,000), and dialyzed against water at $4^{\circ} \mathrm{C}$ to obtain aqueous solution of oxidized CS (oxCS). A certain volume of the remaining solution was freeze-dried, and the amount of the resultant powder was weighed to determine the yield of oxCS.

The volume of the aqueous solution containing $200 \mathrm{mg}$ of oxCS was set at $20 \mathrm{ml}$ by evaporation of and/or addition of water. Then, $\mathrm{mPEG}-\mathrm{NH}_{2}(100 \mathrm{mg})$ was added, and $\mathrm{NaBH}_{3} \mathrm{CN}$ was added gradually, when the $\mathrm{pH}$ of the mixture was adjusted at 6.4-6.6 with $0.1 \mathrm{M} \mathrm{HCl}$ and $0.1 \mathrm{M} \mathrm{NaOH}$. After the mixture was stirred overnight, $\mathrm{NaBH}_{3} \mathrm{CN}(50 \mathrm{mg})$ was further added, and $\mathrm{pH}$ of the mixture was adjusted to 6.4-6.6. After $6 \mathrm{~h}$, the mixture was chromatographed using a Sephadex G50 column in a similar manner. The high molecular weight fractions were gathered and dialyzed against water in a similar manner. Methoxypolyethylene glycol amine-introduced CS (PEG-CS) was obtained as powder by lyophilization of the aqueous solution remaining in the dialysis tube. The chemical structure of PEG-CS (Fig. 1) was investigated by ${ }^{1} \mathrm{H}-\mathrm{NMR}$ measurement, in which the substitution degree was 1:6.7 (mol/mol) of mPEG:(Glc-GalNAc) unit.

Preparation of PEGylated cationic liposomes and siRNA lipoplexes. Cationic liposomes were prepared from DOTAP/DOPE (composition designated as LP-DOTAP), DDAB/DOPE (composition designated as LP-DDAB), and TC-1-12/DOPE (composition designated as LP-TC-1-12), at molar ratios of 1:1. PEGylated cationic liposomes were incorporated with 1, 2, or 3 mol\% PEG-DSPE, PEG-DPPE, PEG-DMPE, PEG-DSG, PEG-DPG, PEG-DMG, or PEG-Chol into each liposomal formulation. 
DDAB

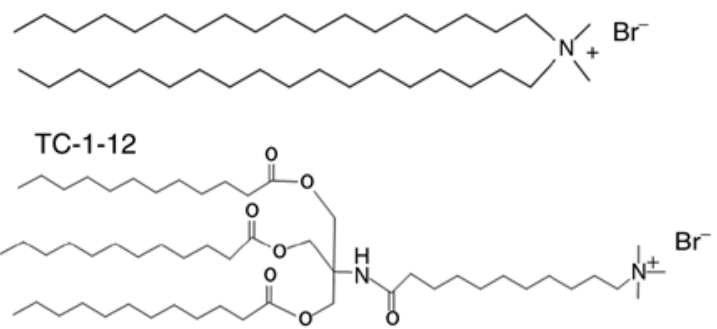

PEG-DSPE

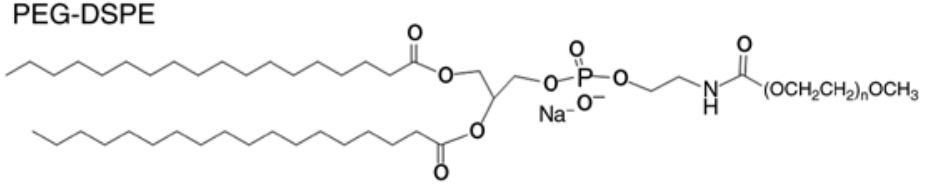

PEG-DPPE

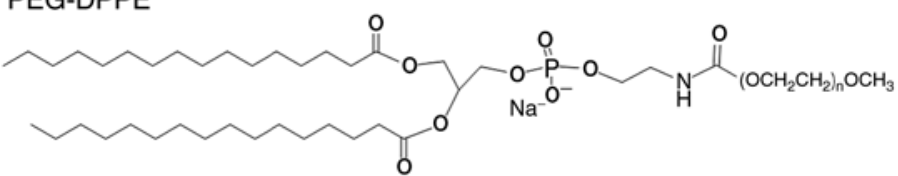

PEG-DMPE

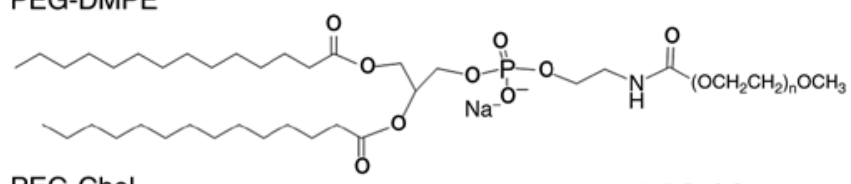

PEG-Chol

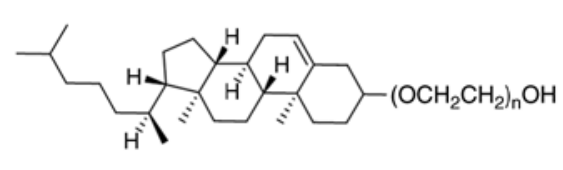

PEG-CS
DOTAP

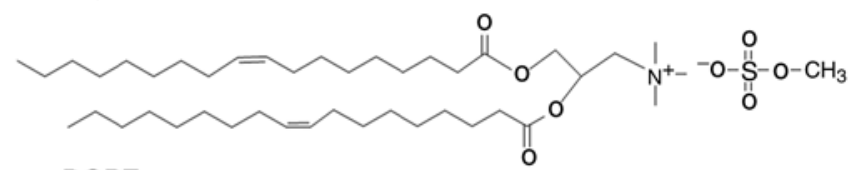

DOPE

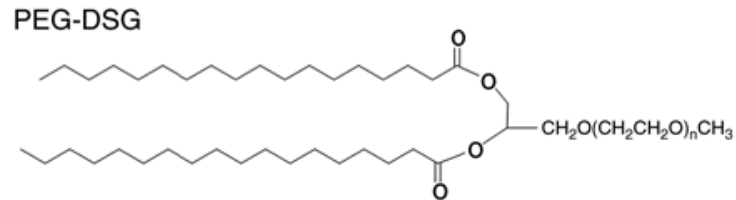

PEG-DPG

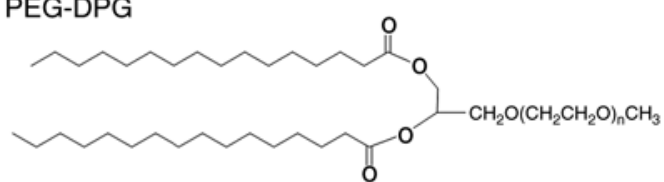

PEG-DMG

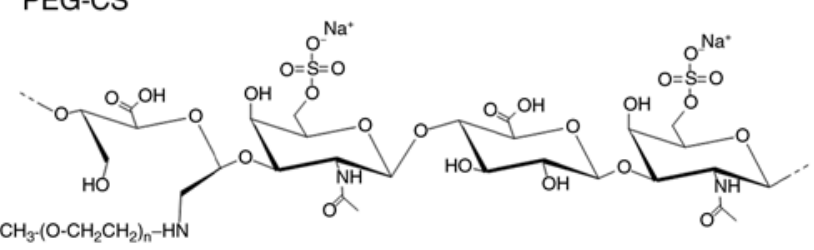

Figure 1. Structure of cationic lipids, neutral lipids and PEG-derivatives ( $\mathrm{n}=45$ for PEG-DSPE, PEG-DPPE, PEG-DMPE, PEG-DSG, PEG-DPG, PEG-DMG, PEG-Chol and PEG-CS). DOTAP, 1,2-dioleoyl-3-trimethylammonium-propane methyl sulfate salt; DDAB, dimethyldioctadecylammonium bromide; TC-1-12, 11-((1,3-bis(dodecanoyloxy)-2-((dodecanoyloxy)methyl)propan-2-yl)amino)- $N, N, N$-trimethyl-11-oxoundecan-1-aminium bromide; DOPE, 1,2-dioleoyl-sn-glycero-3-phosphoethanolamine; PEG-DSPE, $N$-(methylpolyoxyethylene oxycarbonyl)-1,2-distearoyl-sn-glycero-3phosphoethanolamine; PEG-DPPE, $N$-(methylpolyoxyethylene oxycarbonyl)-1,2-dipalmitoyl-sn-glycero-3-phosphoethanolamine sodium salt; PEG-DMPE, $N$-(methylpolyoxyethyleneoxycarbonyl)-1,2-dimyristoyl-sn-glycero-3-phosphoethanolamine sodium salt; PEG-DSG, 1,2-distearoyl-rac-glycero3-methylpolyoxyethylene; PEG-DPG, 1,2-dipalmitoyl-rac-glycero-3-methylpolyoxyethylene; PEG-DMG, 1,2-dimyristoyl-rac-glycero-3-methylpolyoxyethy lene; PEG-Chol, poly(ethylene glycol) cholesteryl ether; PEG-CS, polyoxyethylene-chondroitin sulfate conjugate.

For the preparation of cationic liposomes and PEGylated cationic liposomes using a thin-film hydration method, cationic lipid, DOPE, and PEG-lipid were dissolved in chloroform, and chloroform was evaporated under vacuum in a rotary evaporator at $60^{\circ} \mathrm{C}$ to obtain a thin film. The thin film was hydrated with water at $60^{\circ} \mathrm{C}$ by vortex mixing. The liposomes were sonicated in a bath-type sonicator (Bransonic ${ }^{\circledR}$ 2510J-MTH, $100 \mathrm{~W}$; Branson UL Trasonics Co.) for 5-10 min at room temperature.

To prepare cationic liposome/siRNA complexes (siRNA lipoplexes), each cationic liposome was added to siRNA at a charge ratio (+:-) of 4:1 with vortex-mixing for $10 \mathrm{sec}$ and left at room temperature for $15 \mathrm{~min}$. The theoretical charge ratio (+:-) of cationic liposomes to siRNA is expressed as the molar ratio of nitrogen of cationic lipid to siRNA phosphate.

To prepare siRNA lipoplexes covered with PEG-CS, the cationic liposome suspension was mixed with siRNA by vortex-mixing for $10 \mathrm{~s}$ at a charge ratio (+:-) of 4:1, and left for $15 \mathrm{~min}$ at room temperature. To prepare ternary complexes with PEG-CS, siRNA lipoplexes were mixed with PEG-CS to be 1,
2, or 3 mol\% PEGylation (charge ratios (+:-) of 4:0.9, 4:1.8, and 4:2.7, respectively). The theoretical charge ratio (+:-) of cationic liposomes to CS in PEG-CS was calculated as the molar ratio of nitrogen of cationic lipid to sulfate and carboxylic acid of CS (two negative charges per disaccharide unit).

Size and $\zeta$-potential of PEGylated cationic liposomes and siRNA lipoplexes. The particle size distributions of non-PEGylated and PEGylated cationic liposomes and siRNA lipoplexes were measured by the cumulant method using a light-scattering photometer (ELS-Z2, Otsuka Electronics Co., Ltd.) at $25^{\circ} \mathrm{C}$ after diluting the dispersion to an appropriate volume with water. The $\zeta$-potentials were measured using electrophoresis light-scattering methods with the ELS-Z2 at $25^{\circ} \mathrm{C}$ after diluting the dispersion with an appropriate volume of water.

Accessibility of siRNA in siRNA lipoplexes. The association of siRNAs with cationic liposomes was analyzed using an exclusion assay with $\mathrm{SYBR}^{\circledR}$ Green I Nucleic Acid Gel Stain 
(Takara Bio Inc.) (13). siRNA lipoplexes were formed at charge ratios (+:-) of 1:1, 2:1, 3:1, and 4:1. siRNA lipoplexes with $1 \mu \mathrm{g}$ of siRNA in a volume of $100 \mu \mathrm{l}$ of Tris-HCl buffer ( $\mathrm{pH} 8.0$ ) were mixed with $100 \mu \mathrm{l}$ of 2,500-fold diluted SYBR ${ }^{\circledR}$ Green I Nucleic Acid Gel Stain solution in Tris-HCl buffer, and then incubated for $30 \mathrm{~min}$. Fluorescence was measured at an emission wavelength of $535 \mathrm{~nm}$ with an excitation wavelength of $485 \mathrm{~nm}$ using a fluorescence plate reader (ARVO X2; Perkin Elmer). As a control, the value of fluorescence obtained upon addition of free siRNA solution was set as $100 \%$. The amount of siRNA available to interact with the SYBR ${ }^{\circledR}$ Green I was expressed as a percentage of the control.

Cell culture. Human breast cancer MCF-7 cells stably expressing firefly luciferase (MCF-7-Luc) constructed by transfection of plasmid pcDNA3 containing the firefly luciferase (hLuc) gene from plasmid psiCHECK2 (Promega, Madison, USA) were donated by Dr. Kenji Yamato (University of Tsukuba, Tsukuba, Japan). MCF-7-Luc cells were grown in RPMI-1640 medium, supplemented with $10 \%$ heat-inactivated fetal bovine serum (FBS) and $1.2 \mathrm{mg} / \mathrm{ml} \mathrm{G} 418$ at $37^{\circ} \mathrm{C}$ in a $5 \% \mathrm{CO}_{2}$ humidified atmosphere.

Gene-silencing effects by PEGylated siRNA lipoplexes in cultured cells. MCF-7-Luc cells were seeded in a 6-well culture plate at a density of $3 \times 10^{5}$ cells per well $24 \mathrm{~h}$ prior to transfection. Non-PEGylated and PEGylated siRNA lipoplexes were formed by the addition of non-PEGylated and PEGylated cationic liposomes, respectively, into $50 \mathrm{pmol}$ Cont siRNA or Luc siRNA at a charge ratios (+:-) of 4:1 with vortex-mixing for $10 \mathrm{sec}$, and left at room temperature for $15 \mathrm{~min}$. In PEGylated siRNA lipoplexes with PEG-CS, non-PEGylated lipoplexes with 50 pmol Cont siRNA or Luc siRNA were mixed with PEG-CS at an indicated mol\%, and further left at room temperature for $15 \mathrm{~min}$. For transfection, each siRNA lipoplex was diluted in $1 \mathrm{ml}$ of medium supplemented with $10 \%$ FBS and then the mixture was added to the cells (50 pmol siRNA/well). Forty-eight hours after transfection, the cells were lysed by the addition of $250 \mu 1$ of cell lysis buffer (Pierce ${ }^{\mathrm{TM}}$ Luciferase Cell Lysis Buffer, ThermoFisher Scientific Inc.) after washing with PBS, and subjected to one cycle of freezing $\left(-80^{\circ} \mathrm{C}\right)$ and thawing at $37^{\circ} \mathrm{C}$, followed by centrifugation at $15,000 \mathrm{~g}$ for $10 \mathrm{sec}$. Aliquots of $10 \mu \mathrm{l}$ of the supernatants of cell lysates were mixed with $50 \mu \mathrm{l}$ of PicaGene MelioraStar-LT Luminescence Reagent (Toyo Ink Mfg. Co. Ltd., Tokyo, Japan), and the luminescence was measured as counts per sec (cps) with a chemoluminometer (ARVO X2). Protein concentrations of the supernatants were determined with BCA reagent (Pierce ${ }^{\mathrm{TM}}$ BCA Protein Assay kit; Thermo Fisher Scientific, Inc.), using bovine serum albumin as a standard, and the luciferase activity (cps/ $\mu \mathrm{g}$ protein) was calculated. Luciferase activity (\%) was calculated as relative to the luciferase activity (cps/ $\mu \mathrm{g}$ protein) of untransfected cells.

Cytotoxicity by PEGylated siRNA lipoplexes. MCF-7-Luc cells were seeded in a 96 -well plate $24 \mathrm{~h}$ prior to transfection. Each siRNA lipoplex with 50 pmol Cont siRNA was diluted in $1 \mathrm{ml}$ of medium supplemented with $10 \%$ FBS, and then the mixture (100 $\mu \mathrm{l}$ ) was added to the cells at $50 \%$ confluency in the well (final $50 \mathrm{nM}$ siRNA concentration). After a $24 \mathrm{~h}$ incubation period, cell numbers were determined using a Cell Counting Kit-8 (Dojindo Laboratories). Cell viability was expressed as relative to the absorbance at $450 \mathrm{~nm}$ of untransfected cells.

Agglutination assay. Blood $(0.3 \mathrm{ml})$ was collected from the jugular vein of one female BALB/c mice ( 8 weeks of age; Sankyo Labo Service Corp. Inc.) while under anesthesia by an intraperitoneal injection of $50 \mathrm{mg} / \mathrm{kg}$ body weight of pentobarbital (Nembutal, Dainippon Pharmaceutical Co., Ltd.). Erythrocytes were collected from the whole blood at $4^{\circ} \mathrm{C}$ by centrifugation at $300 \mathrm{~g}$ for $3 \mathrm{~min}$ and resuspended in PBS as a $2 \%(\mathrm{v} / \mathrm{v})$ suspension of erythrocytes. Non-PEGylated and PEGylated siRNA lipoplexes with $2 \mu \mathrm{g}$ siRNA were added to $100 \mu \mathrm{l}$ of $2 \%$ (v/v) erythrocyte suspension, respectively. After incubation for $15 \mathrm{~min}$ at $37^{\circ} \mathrm{C}$, the sample was placed on a glass plate and agglutination was observed by microscopy.

Biodistribution of siRNA after intravenous injection of PEGylated siRNA lipoplexes into mice. A total of 43 female BALB/c mice (18-20 g, 8 weeks of age; Sankyo Labo Service Corp. Inc.) were housed in a temperature $\left(24^{\circ} \mathrm{C}\right)$ and humidity (55\%) controlled room with a $12 \mathrm{~h}$ light/dark cycle (lights on at 8:00 a.m.) with ad libitum access to food and water. For all experiments, health and behavior of the mice were checked daily. Non-PEGylated and PEGylated siRNA lipoplexes were formed by the addition of non-PEGylated and PEGylated cationic liposomes, respectively, into $20 \mu \mathrm{g}$ of Cy5.5-siRNA with vortex mixing for $10 \mathrm{sec}$ and left at room temperature for 15 min. In PEGylated siRNA lipoplexes with PEG-CS, non-PEGylated lipoplexes with $20 \mu \mathrm{g}$ of Cy5.5-siRNA were mixed with PEG-CS at an indicated mol\%, and further left at room temperature for $15 \mathrm{~min}$. The non-PEGylated or PEGylated siRNA lipoplexes were administered intravenously via the lateral tail vein into female BALB/c mice $(n=1$ for each siRNA lipoplex). Based on our previous study (18), mice were sacrificed at $1 \mathrm{~h}$ after intravenous injection of siRNA lipoplexes into mice (pre-defined endpoint). One hour after injection, mice were sacrificed by cervical dislocation (a total of 43 mice), and euthanasia after cervical dislocation was confirmed by careful assessment of the mice for unambiguous signs of death such as cardiac arrest. Cy5.5 fluorescence imaging of the tissues was performed using a NightOWL LB981 NC100 system (Berthold Technologies). In Cy5.5 fluorescence imaging, the excitation and emission filters were set at 630/20 and 680/30 nm, respectively. The exposure time for fluorescence was $5 \mathrm{sec}$. A grayscale body-surface reference image was collected using a NightOWL LB981 CCD camera. The images were analyzed using IndiGo2 software (version 2.0.1.0) provided with the in vivo imaging system (Berthold Technologies). The tissues after fluorescence imaging were frozen on dry ice and sliced into $16-\mu \mathrm{m}$ sections. The localization of Cy5.5-siRNA was examined using an Eclipse TS100-F microscope (Nikon).

Statistical analysis. Data are presented as the mean + standard deviation (SD) of triple determinations. The statistical significance of differences between mean values was determined by Student's t-test using GraphPad Prism 4.0 (GraphPad Software Inc.). Multiple measurement comparisons were performed by analysis of variance followed by one-way analysis of variance on ranks with post hoc Tukey test using 
Table I. Particle size and $\zeta$-potential of DDAB-based liposomes and lipoplexes of small interfering RNA.

\begin{tabular}{|c|c|c|c|c|c|c|}
\hline \multirow[b]{2}{*}{ Liposome } & \multicolumn{3}{|c|}{ Liposomes } & \multicolumn{3}{|c|}{ Lipoplexes $^{\mathrm{b}}$} \\
\hline & $\operatorname{Size}^{\mathrm{a}}(\mathrm{nm})$ & PDI & $\zeta$-potential ${ }^{\mathrm{a}}(\mathrm{mV})$ & $\operatorname{Size}^{\mathrm{a}}(\mathrm{nm})$ & PDI & $\zeta$-potential ${ }^{\mathrm{a}}(\mathrm{mV})$ \\
\hline LP-DDAB & $104.2 \pm 0.8$ & $0.21 \pm 0.01$ & $48.0 \pm 3.6$ & $177.3 \pm 1.8$ & $0.17 \pm 0.02$ & $43.1 \pm 1.0$ \\
\hline LP-DDAB-PEG-DSPE (1 mol\%) & $100.5 \pm 0.6$ & $0.23 \pm 0.01$ & $49.5 \pm 0.3$ & $198.0 \pm 1.4$ & $0.22 \pm 0.01$ & $37.3 \pm 0.7$ \\
\hline LP-DDAB-PEG-DSPE (2 mol\%) & $103.3 \pm 0.3$ & $0.24 \pm 0.01$ & $52.5 \pm 3.1$ & $180.1 \pm 0.9$ & $0.19 \pm 0.01$ & $33.4 \pm 0.4$ \\
\hline LP-DDAB-PEG-DSPE (3 mol\%) & $105.9 \pm 1.2$ & $0.23 \pm 0.01$ & $43.5 \pm 4.1$ & $224.1 \pm 2.5$ & $0.26 \pm 0.01$ & $28.7 \pm 0.6$ \\
\hline LP-DDAB-PEG-DPPE (1 mol\%) & $107.2 \pm 0.9$ & $0.26 \pm 0.01$ & $45.3 \pm 0.7$ & $151.6 \pm 1.4$ & $0.19 \pm 0.01$ & $42.2 \pm 1.9$ \\
\hline LP-DDAB-PEG-DPPE (2 mol\%) & $95.5 \pm 1.1$ & $0.24 \pm 0.01$ & $43.6 \pm 0.2$ & $160.4 \pm 2.1$ & $0.18 \pm 0.01$ & $38.9 \pm 0.9$ \\
\hline LP-DDAB-PEG-DPPE (3 mol\%) & $94.0 \pm 0.8$ & $0.24 \pm 0.01$ & $43.1 \pm 0.9$ & $170.5 \pm 3.1$ & $0.19 \pm 0.02$ & $38.0 \pm 0.2$ \\
\hline LP-DDAB-PEG-DMPE (1 mol\%) & $104.3 \pm 1.0$ & $0.26 \pm 0.00$ & $50.4 \pm 0.6$ & $189.0 \pm 2.6$ & $0.21 \pm 0.01$ & $42.2 \pm 0.3$ \\
\hline LP-DDAB-PEG-DMPE (2 mol\%) & $124.5 \pm 3.5$ & $0.27 \pm 0.00$ & $49.3 \pm 0.8$ & $187.2 \pm 4.0$ & $0.23 \pm 0.01$ & $39.4 \pm 0.5$ \\
\hline LP-DDAB-PEG-DMPE (3 mol\%) & $114.5 \pm 0.8$ & $0.29 \pm 0.01$ & $48.6 \pm 0.8$ & $171.0 \pm 3.2$ & $0.22 \pm 0.01$ & $37.9 \pm 0.5$ \\
\hline LP-DDAB-PEG-DSG (1 mol\%) & $96.6 \pm 2.2$ & $0.23 \pm 0.01$ & $38.0 \pm 0.8$ & $178.1 \pm 0.8$ & $0.21 \pm 0.01$ & $39.3 \pm 1.6$ \\
\hline LP-DDAB-PEG-DSG (2 mol\%) & $94.2 \pm 0.6$ & $0.22 \pm 0.00$ & $41.9 \pm 3.1$ & $188.8 \pm 3.4$ & $0.23 \pm 0.02$ & $35.0 \pm 0.9$ \\
\hline LP-DDAB-PEG-DSG (3 mol\%) & $95.1 \pm 2.5$ & $0.24 \pm 0.01$ & $45.7 \pm 1.5$ & $179.9 \pm 3.5$ & $0.22 \pm 0.01$ & $35.1 \pm 0.9$ \\
\hline LP-DDAB-PEG-DPG (1 mol\%) & $104.0 \pm 1.3$ & $0.25 \pm 0.01$ & $48.5 \pm 0.9$ & $211.8 \pm 1.4$ & $0.22 \pm 0.01$ & $42.1 \pm 0.6$ \\
\hline LP-DDAB-PEG-DPG (2 mol\%) & $113.9 \pm 0.9$ & $0.25 \pm 0.01$ & $48.3 \pm 0.4$ & $209.8 \pm 4.3$ & $0.25 \pm 0.01$ & $36.7 \pm 2.3$ \\
\hline LP-DDAB-PEG-DPG (3 mol\%) & $109.0 \pm 1.3$ & $0.25 \pm 0.01$ & $50.1 \pm 3.7$ & $184.6 \pm 3.1$ & $0.26 \pm 0.01$ & $34.3 \pm 0.7$ \\
\hline LP-DDAB-PEG-DMG (1 mol\%) & $116.5 \pm 0.7$ & $0.25 \pm 0.01$ & $52.3 \pm 0.9$ & $192.9 \pm 1.9$ & $0.20 \pm 0.01$ & $39.8 \pm 0.4$ \\
\hline LP-DDAB-PEG-DMG (2 mol\%) & $93.7 \pm 0.7$ & $0.23 \pm 0.00$ & $52.8 \pm 0.4$ & $234.6 \pm 3.3$ & $0.24 \pm 0.01$ & $37.8 \pm 0.4$ \\
\hline LP-DDAB-PEG-DMG (3 mol\%) & $116.7 \pm 0.7$ & $0.25 \pm 0.00$ & $50.2 \pm 0.7$ & $180.5 \pm 0.9$ & $0.23 \pm 0.01$ & $34.6 \pm 0.3$ \\
\hline LP-DDAB-PEG-Chol (1 mol\%) & $102.1 \pm 1.1$ & $0.21 \pm 0.01$ & $47.6 \pm 3.1$ & $196.2 \pm 4.2$ & $0.23 \pm 0.01$ & $48.3 \pm 1.0$ \\
\hline LP-DDAB-PEG-Chol (2 mol\%) & $108.1 \pm 2.6$ & $0.15 \pm 0.02$ & $42.7 \pm 1.6$ & $177.8 \pm 3.2$ & $0.19 \pm 0.01$ & $39.7 \pm 1.0$ \\
\hline LP-DDAB-PEG-Chol (3 mol\%) & $95.4 \pm 1.2$ & $0.22 \pm 0.02$ & $43.4 \pm 2.2$ & $191.6 \pm 5.3$ & $0.21 \pm 0.01$ & $35.3 \pm 0.9$ \\
\hline
\end{tabular}

${ }^{\text {an }}$ water. ${ }^{b}$ Charge ratio (+:-) of cationic lipid to siRNA phosphate $=4: 1$. Data are presented as the mean \pm SD ( $=3$ ). DDAB, Dimethyldioctadecylammonium bromide; LP, liposome; PEG, polyethylene glycol; PDI, polydispersity index.

GraphPad Prism 4.0. $\mathrm{P}<0.05$ was considered to indicate a statistically significant difference.

\section{Results and Discussion}

Characterization of cationic liposomes and siRNA lipoplexes. For the PEGylation of liposomes, a commonly used lipid derivative of PEG is PEG-DSPE, and PEG-DSPE is incorporated into the liposomal membrane via the DSPE anchor during the preparation of cationic liposomes. In this study, first, to examine whether the anchor of PEG-lipid derivatives in PEGylated siRNA lipoplexes affected in vitro gene silencing, we prepared PEGylated cationic liposomes with various types of PEG-lipid derivatives. Here, we used DDAB and DOTAP as dialkyl cationic lipids; and TC-1-12 as a trialkyl cationic lipid (Fig. 1). For the preparation of non-PEGylated cationic liposomes, LP-DDAB, LP-DOTAP, and LP-TC-1-12 were prepared from DDAB/DOPE, DOTAP/DOPE, and TC-1-12/DOPE, respectively, at a molar ratio of 1:1. For the preparation of PEGylated cationic liposomes, 1, 2, or $3 \mathrm{~mol} \%$ PEG-DSPE, PEG-DPPE, PEG-DMPE, PEG-DSG, PEG-DPG, PEG-DMG, and PEG-Chol were included into the formulations of cationic liposomes. For example, LP-DDAB-PEG-DSPE, LP-DDAB-PEG-DPPE, LP-DDAB-PEG-DMPE, LP-DDABPEG-DSG, LP-DDAB-PEG-DPG, LP-DDAB-PEG-DMG, and LP-DDAB-PEG-Chol included PEG-DSPE, PEG-DPPE, PEG-DMPE, PEG-DSG, PEG-DPG, PEG-DMG, and PEG-Chol, respectively, at an indicated mol\% into the formulation of LP-DDAB (Table I). The sizes of PEGylated cationic liposomes were approximately 90-120 nm, and the $\zeta$-potentials were approximately $+37-53 \mathrm{mV}$ (Tables I-III). When the liposomes were mixed with siRNA, the lipoplex sizes were approximately $150-310 \mathrm{~nm}$ and their $\zeta$-potentials were approximately +29-48 mV (Tables I-III).

Previously, we developed anionic polymer-coated siRNA lipoplexes and found that chondroitin sulfate (CS) coatings for siRNA lipoplexes produced safe systemic vectors $(19,20)$. Therefore, for the development of PEG-coating via electrostatic interaction between positively charged siRNA lipoplexes and negatively charged CS, we synthesized PEG-chondroitin sulfate conjugate (PEG-CS). CS consists of linear repeating disaccharide units of glucuronic acid and sulfated galactosamine, and PEG-CS has linear PEG $_{2000}$ molecules introduced onto glucuronic acid of CS in a $\mathrm{PEG}_{2000}$ molecule per approximately 6-7 disaccharide units of CS (Fig. 1). For the preparation of siRNA lipoplexes coated with PEG-CS, LP-DOTAP, LP-DDAB, and LP-TC-1-12 were mixed with siRNA a charge ratio (+:-) of 4:1, and then PEG-CS solution was added to these siRNA lipoplexes at 1, 2, or 3 mol\% PEG in PEG-CS in the formulation of cationic liposomes. The $\zeta$-potentials 
Table II. Particle size and $\zeta$-potential of DOTAP-based liposomes and lipoplexes of siRNA.

\begin{tabular}{|c|c|c|c|c|c|c|}
\hline \multirow[b]{2}{*}{ Liposome } & \multicolumn{3}{|c|}{ Liposomes } & \multicolumn{3}{|c|}{ Lipoplexes $^{\mathrm{b}}$} \\
\hline & $\operatorname{Size}^{\mathrm{a}}(\mathrm{nm})$ & PDI & $\zeta$-potential ${ }^{\mathrm{a}}(\mathrm{mV})$ & $\operatorname{Size}^{\mathrm{a}}(\mathrm{nm})$ & PDI & $\zeta$-potential ${ }^{\mathrm{a}}(\mathrm{mV})$ \\
\hline LP-DOTAP & $105.0 \pm 0.5$ & $0.19 \pm 0.01$ & $44.0 \pm 6.1$ & $160.9 \pm 0.7$ & $0.18 \pm 0.01$ & $38.1 \pm 0.8$ \\
\hline LP-DOTAP-PEG-DSPE (1 mol\%) & $108.8 \pm 0.7$ & $0.24 \pm 0.01$ & $46.5 \pm 7.9$ & $179.4 \pm 1.5$ & $0.24 \pm 0.02$ & $40.5 \pm 1.6$ \\
\hline LP-DOTAP-PEG-DSPE (2 mol\%) & $88.7 \pm 2.0$ & $0.26 \pm 0.01$ & $40.5 \pm 0.6$ & $158.1 \pm 2.9$ & $0.24 \pm 0.01$ & $41.2 \pm 0.6$ \\
\hline LP-DOTAP-PEG-DSPE (3 mol\%) & $114.5 \pm 2.3$ & $0.28 \pm 0.02$ & $37.9 \pm 0.4$ & $174.3 \pm 2.7$ & $0.27 \pm 0.00$ & $37.1 \pm 1.3$ \\
\hline LP-DOTAP-PEG-DSG (1 mol\%) & $113.7 \pm 0.4$ & $0.25 \pm 0.01$ & $49.7 \pm 1.2$ & $171.1 \pm 3.3$ & $0.23 \pm 0.01$ & $32.2 \pm 1.4$ \\
\hline LP-DOTAP-PEG-DSG (2 mol\%) & $118.6 \pm 1.6$ & $0.26 \pm 0.00$ & $39.3 \pm 0.5$ & $174.2 \pm 3.0$ & $0.26 \pm 0.01$ & $40.2 \pm 0.5$ \\
\hline LP-DOTAP-PEG-DSG (3 mol\%) & $117.7 \pm 1.2$ & $0.25 \pm 0.01$ & $40.4 \pm 0.6$ & $155.7 \pm 2.8$ & $0.26 \pm 0.01$ & $40.2 \pm 0.4$ \\
\hline LP-DOTAP-PEG-Chol (1 mol\%) & $111.2 \pm 12.9$ & $0.16 \pm 0.13$ & $37.3 \pm 2.2$ & $181.8 \pm 1.3$ & $0.23 \pm 0.02$ & $35.9 \pm 2.1$ \\
\hline LP-DOTAP-PEG-Chol (2 mol\%) & $112.3 \pm 4.5$ & $0.26 \pm 0.02$ & $41.2 \pm 1.7$ & $170.4 \pm 0.4$ & $0.20 \pm 0.01$ & $39.4 \pm 0.7$ \\
\hline LP-DOTAP-PEG-Chol (3 mol\%) & $92.8 \pm 0.5$ & $0.21 \pm 0.01$ & $40.1 \pm 3.9$ & $162.0 \pm 3.8$ & $0.26 \pm 0.04$ & $34.2 \pm 1.9$ \\
\hline
\end{tabular}

${ }^{a}$ In water. ${ }^{b}$ Charge ratio (+:-) of cationic lipid to siRNA phosphate $=4: 1$. Data are presented as the mean \pm SD ( $=3$ ). DOTAP, 1,2-Dioleoyl-3-trimethylammonium-propane methyl sulfate salt; siRNA, small interfering RNA; LP, liposome; PEG, polyethylene glycol; PDI, polydispersity index.

Table III. Particle size and $\zeta$-potential of TC-1-12-based liposomes and lipoplexes of siRNA.

\begin{tabular}{|c|c|c|c|c|c|c|}
\hline \multirow[b]{2}{*}{ Liposome } & \multicolumn{3}{|c|}{ Liposomes } & \multicolumn{3}{|c|}{ Lipoplexes $^{\mathrm{b}}$} \\
\hline & $\operatorname{Size}^{\mathrm{a}}(\mathrm{nm})$ & PDI & $\zeta$-potential ${ }^{\mathrm{a}}(\mathrm{mV})$ & $\operatorname{Size}^{\mathrm{a}}(\mathrm{nm})$ & PDI & $\zeta$-potential ${ }^{\mathrm{a}}(\mathrm{mV})$ \\
\hline LP-TC-1-12 & $114.9 \pm 0.9$ & $0.24 \pm 0.01$ & $46.6 \pm 0.6$ & $168.6 \pm 2.8$ & $0.18 \pm 0.01$ & $41.0 \pm 0.9$ \\
\hline LP-TC-1-12-PEG-DSPE (1 mol\%) & $98.6 \pm 1.1$ & $0.24 \pm 0.01$ & $44.6 \pm 1.0$ & $314.2 \pm 16.3$ & $0.31 \pm 0.01$ & $37.3 \pm 0.4$ \\
\hline LP-TC-1-12-PEG-DSPE (2 mol\%) & $96.2 \pm 1.8$ & $0.23 \pm 0.00$ & $38.5 \pm 3.8$ & $184.3 \pm 2.9$ & $0.27 \pm 0.01$ & $39.1 \pm 0.4$ \\
\hline LP-TC-1-12-PEG-DSPE (3 mol\%) & $90.9 \pm 2.2$ & $0.26 \pm 0.02$ & $48.4 \pm 0.9$ & $164.0 \pm 1.5$ & $0.21 \pm 0.01$ & $34.8 \pm 0.2$ \\
\hline LP-TC-1-12-PEG-DSG (1 mol\%) & $88.0 \pm 0.4$ & $0.25 \pm 0.01$ & $40.1 \pm 3.4$ & $138.9 \pm 1.0$ & $0.13 \pm 0.01$ & $40.1 \pm 0.7$ \\
\hline LP-TC-1-12-PEG-DSG (2 mol\%) & $107.9 \pm 1.8$ & $0.24 \pm 0.00$ & $40.4 \pm 1.8$ & $157.4 \pm 0.8$ & $0.15 \pm 0.02$ & $35.9 \pm 0.5$ \\
\hline LP-TC-1-12-PEG-DSG (3 mol\%) & $91.1 \pm 0.6$ & $0.24 \pm 0.00$ & $50.6 \pm 1.4$ & $151.7 \pm 2.2$ & $0.16 \pm 0.00$ & $42.2 \pm 1.3$ \\
\hline LP-TC-1-12-PEG-Chol (1 mol\%) & $110.7 \pm 2.0$ & $0.22 \pm 0.02$ & $37.9 \pm 2.2$ & $180.0 \pm 4.2$ & $0.18 \pm 0.02$ & $37.1 \pm 0.5$ \\
\hline LP-TC-1-12-PEG-Chol (2 mol\%) & $118.0 \pm 1.0$ & $0.25 \pm 0.00$ & $40.8 \pm 1.6$ & $182.9 \pm 2.5$ & $0.16 \pm 0.01$ & $34.6 \pm 0.8$ \\
\hline LP-TC-1-12-PEG-Chol (3 mol\%) & $100.7 \pm 1.0$ & $0.23 \pm 0.01$ & $47.2 \pm 0.7$ & $169.6 \pm 2.2$ & $0.16 \pm 0.02$ & $34.0 \pm 1.0$ \\
\hline
\end{tabular}

${ }^{a}$ In water. ${ }^{b}$ Charge ratio (+:-) of cationic lipid to siRNA phosphate $=4: 1$. Data are presented as the mean $\pm \mathrm{SD}(\mathrm{n}=3$ ). TC-1-12, 11-((1,3-bis(dodecanoyloxy)-2-((dodecanoyloxy)methyl)propan-2-yl)amino)- $N, N, N$-trimethyl-11-oxoundecan-1-aminium bromide; siRNA, small interfering RNA; LP, liposome; PEG, polyethylene glycol; PDI, polydispersity index.

of the ternary complexes after the addition of PEG-CS were almost consistently negative around 2 mol\% PEG in PEG-CS (Table IV), indicating that nitrogen of the siRNA lipoplex was completely covered with a sulfate group or a carboxyl group of CS. The sizes of PEGylated siRNA lipoplexes with PEG-CS were approximately 160-250 $\mathrm{nm}$ regardless of the cationic lipid type in the cationic liposomes.

Next, we examined the association of siRNA with each PEGylated cationic liposome using an exclusion assay with SYBR $^{\circledR}$ Green I. SYBR ${ }^{\circledR}$ Green I is a DNA/RNA-intercalating agent whose fluorescence is dramatically enhanced upon binding to unbound siRNA in cationic liposome suspension. As a result, in DDAB- and DOTAP-based PEGylated cationic liposomes, the fluorescence of SYBR ${ }^{\circledR}$ Green I was markedly decreased by the addition of PEGylated cationic liposomes into the siRNA solution above charge ratios (+:-) of 2:1, compared with that in siRNA solution (Fig. 2). This result suggested that siRNAs were completely bound to each cationic liposome regardless of the type of PEG-lipid derivatives in DDAB- and DOTAP-based PEGylated cationic liposomes. In contrast, in TC-1-12-based PEGylated cationic liposomes, the decreases in fluorescence after the addition of PEGylated cationic liposomes into the siRNA solution were saturated at around $20-50 \%$ of free siRNA, indicating that PEGylation in LP-TC-1-12 may partially inhibit the interaction between siRNA and cationic liposomes. Furthermore, in PEGylated siRNA lipoplexes with PEG-CS, an increase in unbound siRNA $(\sim 30 \%)$ was observed with increasing 
Table IV. Particle size and $\zeta$-potential of PEG-CS coated siRNA lipoplexes.

\begin{tabular}{lcccc}
\hline Lipoplex & Charge ratio $^{\mathrm{a}}\left(+:-\mathbf{-}^{-}\right)$ & Size $^{\mathrm{b}}(\mathrm{nm})$ & PDI & $\zeta$-potential $^{\mathrm{b}}(\mathrm{mV})$ \\
\hline LP-DOTAP-PEG-CS $(1 \mathrm{~mol} \%)$ & $4: 1: 0.9$ & $250.0 \pm 1.7$ & $0.20 \pm 0.01$ & $39.7 \pm 0.1$ \\
LP-DOTAP-PEG-CS (2 mol\%) & $4: 1: 1.8$ & $192.5 \pm 2.3$ & $0.15 \pm 0.03$ & $-26.0 \pm 0.5$ \\
LP-DOTAP-PEG-CS (3 mol\%) & $4: 1: 2.7$ & $183.6 \pm 2.1$ & $0.12 \pm 0.00$ & $-32.4 \pm 1.1$ \\
LP-DDAB-PEG-CS (1 mol\%) & $4: 1: 0.9$ & $211.0 \pm 0.9$ & $0.11 \pm 0.01$ & $41.6 \pm 0.5$ \\
LP-DDAB-PEG-CS (2 mol\%) & $4: 1: 1.8$ & $165.8 \pm 0.6$ & $0.12 \pm 0.01$ & $-28.9 \pm 1.2$ \\
LP-DDAB-PEG-CS (3 mol\%) & $4: 1: 2.7$ & $166.8 \pm 1.7$ & $0.12 \pm 0.01$ & $-39.2 \pm 1.0$ \\
LP-TC-1-12-PEG-CS (1 mol\%) & $4: 1: 0.9$ & $168.6 \pm 1.5$ & $0.13 \pm 0.02$ & $27.0 \pm 1.8$ \\
LP-TC-1-12-PEG-CS (2 mol\%) & $4: 1: 1.8$ & $212.1 \pm 41.1$ & $0.10 \pm 0.02$ & $-28.3 \pm 1.9$ \\
LP-TC-1-12-PEG-CS (3 mol\%) & $4: 1: 2.7$ & $161.6 \pm 1.5$ & $0.10 \pm 0.02$ & $-26.9 \pm 1.1$
\end{tabular}

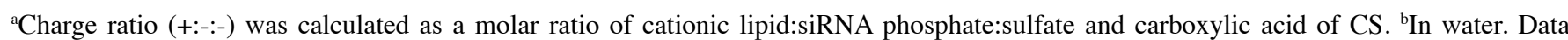
are presented as the mean \pm SD $(n=3)$. PEG, polyethylene glycol; CS, chondroitin sulfate C sodium salt; siRNA, small interfering RNA; LP, liposome; PDI, polydispersity index.
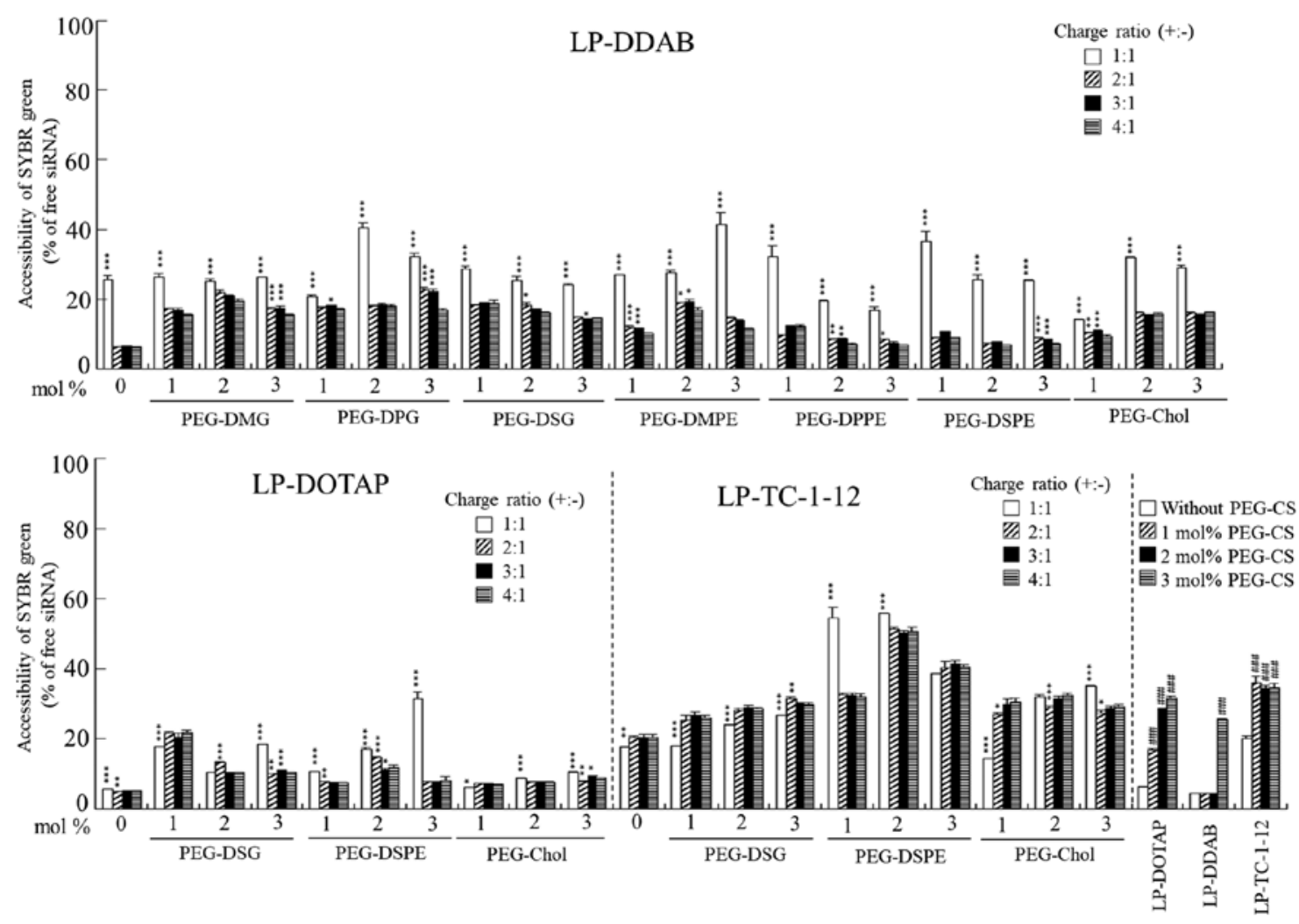

Figure 2. Association of siRNA with PEGylated cationic liposomes in an exclusion assay using SYBR ${ }^{\circledR}$ Green I Nucleic Acid Gel Stain. PEGylated siRNA lipoplexes were prepared by mixing siRNA with 1, 2 or 3 mol\% PEGylated cationic liposomes at charge ratios (+:-) of 1:1, 2:1, 3:1 and 4:1, respectively. In PEGylated siRNA lipoplexes with PEG-CS, siRNA lipoplexes were prepared by mixing siRNA with cationic liposomes at a charge ratio (+:-) of 4:1. PEG-CS was subsequently added at 1,2 or $3 \mathrm{~mol} \%$ PEGylation. As a control, the value of fluorescence obtained after the addition of free siRNA solution was set as $100 \%$. The amount of siRNA available to interact with $\mathrm{SYBR}^{\circledast}$ Green I is expressed as a percentage of the control. Each column represents the mean $+\mathrm{SD}$ $(\mathrm{n}=3) .{ }^{*} \mathrm{P}<0.05,{ }^{* *} \mathrm{P}<0.01$ and ${ }^{* * * *} \mathrm{P}<0.001$ vs. a charge ratio (+:-) of 4:1 in PEGylated siRNA liposomes with PEG-DMG, PEG-DPG, PEG-DSG, PEG-DMPE, PEG-DPPE, PEG-DSPE and PEG-Chol. ${ }^{\# \# \# ~} \mathrm{P}<0.001$ vs. non-PEGylated siRNA lipoplexes in PEGylated siRNA lipoplexes with PEG-CS. siRNA, small interfering RNA; PEG, polyethylene glycol; LP-DDAB, DDAB liposome; LP-DOTAP, DOTAP liposome; LP-TC-1-12, TC-1-12 liposome.

amounts of PEG-CS added to the siRNA lipoplexes, suggesting that some siRNA molecules may be released from the siRNA lipoplexes by the addition of negatively charged PEG-CS.

Effects of anchor type of the PEG derivative in PEGylated siRNA lipoplexes on in vitro gene knockdown efficacy. To examine the effects of the PEG anchor in PEGylated siRNA lipoplexes on gene knockdown, MCF-7-Luc cells were incubated with 1, 2, or 3 mol\% PEGylated siRNA lipoplexes at a final concentration of $50 \mathrm{nM}$ siRNA, and the gene-silencing effects were assessed by assaying luciferase activity. Non-PEGylated LP-DOTAP, LP-DDAB, and LP-TC-1-12 

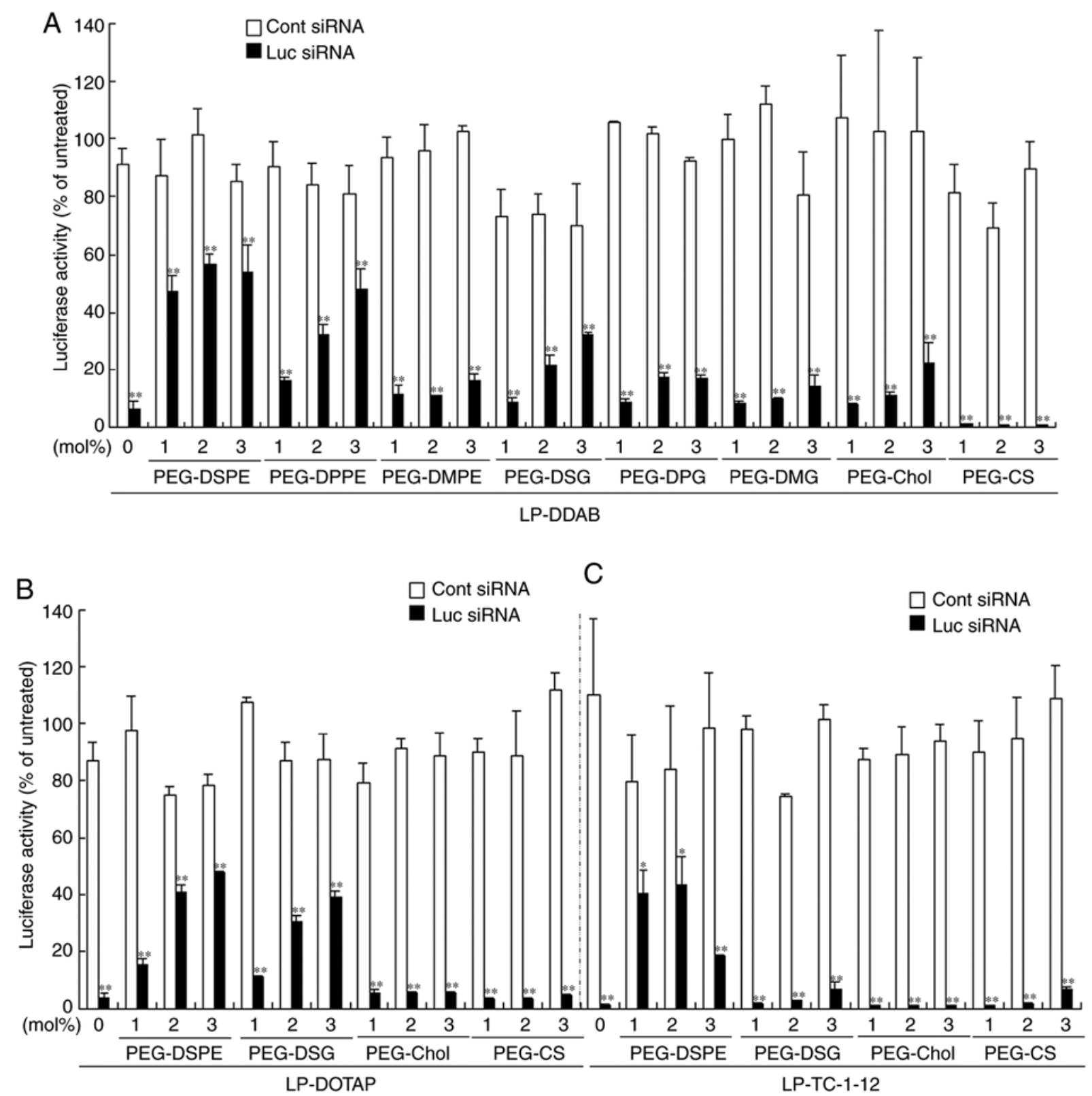

Figure 3. Effects of PEGylated cationic liposomes on gene suppression in MCF-7-Luc cells after transfection with siRNA lipoplexes. (A) DDAB, (B) DOTAP or (C) TC-1-12 based siRNA lipoplexes modified with 1,2 and 3 mol\% PEG-lipid derivative or PEG-CS were added to MCF-7-Luc cells (50 nM siRNA). Luciferase assays were carried out after incubation for $48 \mathrm{~h}$. Each column represents the mean $+\mathrm{SD}(\mathrm{n}=3)$. ${ }^{*} \mathrm{P}<0.05$ and ${ }^{* * *} \mathrm{P}<0.01$ vs. Cont siRNA. PEG, polyethylene glycol; siRNA, small interfering RNA; LP-DDAB, DDAB liposome; LP-DOTAP, DOTAP liposome; LP-TC-1-12, TC-1-12 liposome; Cont, control.

lipoplexes with Luc siRNA strongly suppressed luciferase activity (Fig. 3). In DDAB-based PEGylated siRNA lipoplexes, LP-DDAB-PEG-DSPE and LP-DDAB-PEG-DPPE lipoplexes strongly decreased the gene-silencing activity with increasing amounts of PEG-DSPE and PEG-DPPE, respectively, and LP-DDAB-PEG-DSG moderately with increasing amounts of PEG-DSG. However, LP-DDAB-PEG-DMPE, LP-DDAB-PEG-DPG, LP-DDAB-PEG-DMG, LP-DDABPEG-Chol, and LP-DDAB-PEG-CS lipoplexes did not greatly affect the gene-silencing effects by PEGylation, compared with non-PEGylated LP-DDAB lipoplexes (Fig. 3A). PEGylation with PEG-lipids with long dialkyl chains (C16-C18) and a phosphate group trended to inhibit the gene-silencing effects in the cells by siRNA lipoplexes, indicating that PEG-lipids with short dialkyl chains and/or without phosphate groups may be easily detached from PEGylated siRNA lipoplexes in culture medium. In DOTAP-based PEGylated siRNA lipoplexes, LP-DOTAP-PEG-DSPE and LP-DOTAP-PEG-DSG lipoplexes decreased the gene-silencing activity with increasing amounts of PEG-DSPE and PEG-DSG, respectively; however, LP-DOTAP-PEG-Chol and LP-DOTAP-PEG-CS lipoplexes did not show an affect by PEGylation, compared with non-PEGylated LP-DOTAP lipoplexes (Fig. 3B). Furthermore, in TC-1-12-based PEGylated siRNA lipoplexes, LP-TC-1-12-PEG-DSPE lipoplexes decreased the gene-silencing activity with increasing amounts of PEG-DSPE; however, LP-TC-1-12-DSG, LP-TC-1-12-PEG-Chol, and LP-TC-1-12-PEG-CS lipoplexes did not show an affect by PEGylation, compared with non-PEGylated LP-TC-1-12 lipoplexes (Fig. 3C). As a result, PEGylation of siRNA 

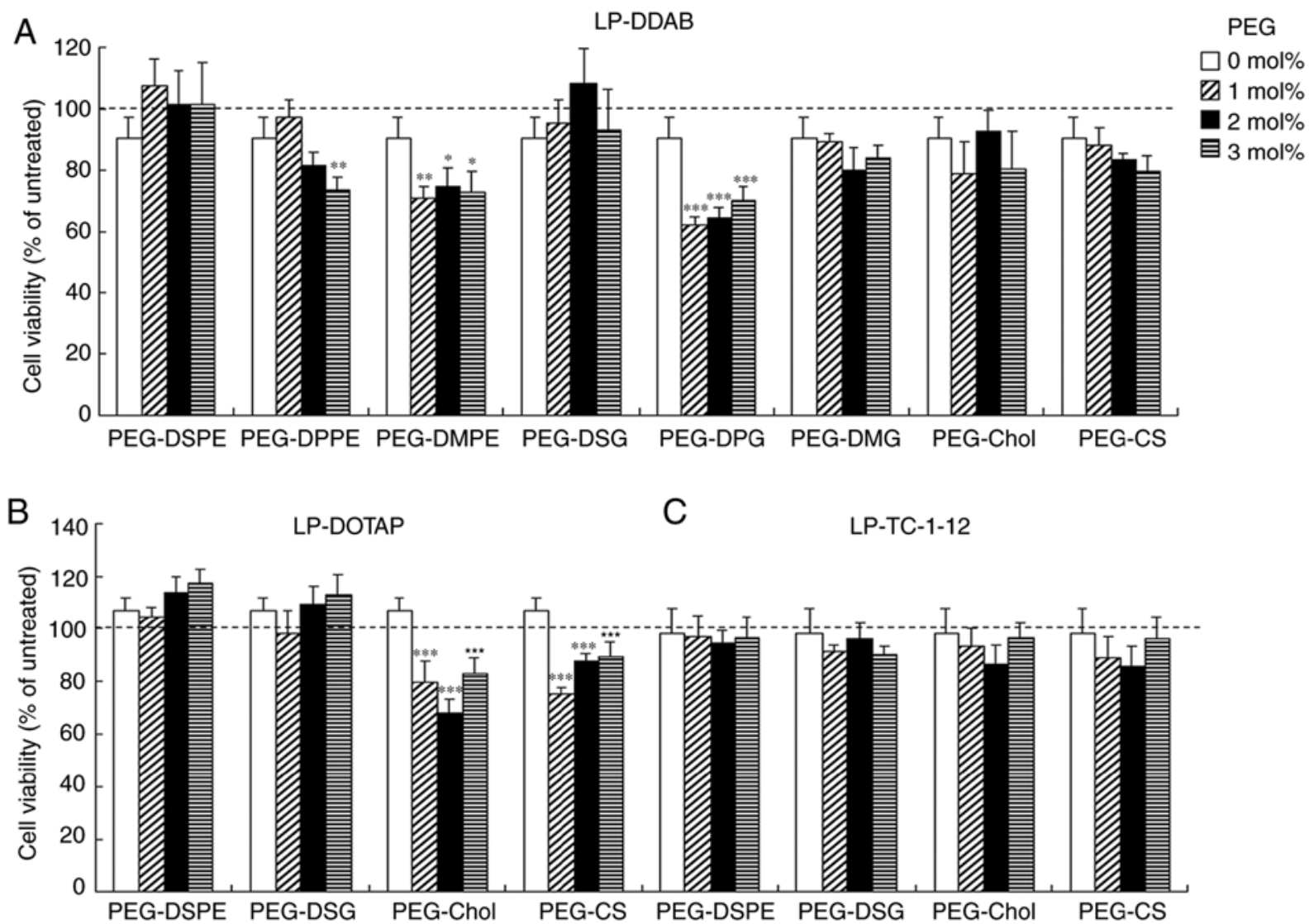

Figure 4. MCF-7-Luc cell viability was detected $24 \mathrm{~h}$ after transfection with PEGylated siRNA lipoplexes. Non-PEGylated and 1, 2 or 3 mol\% PEGylated siRNA lipoplexes were added to MCF-7-Luc cells (50 nM siRNA). (A) LP-DDAB lipoplexes, (B) LP-DOTAP lipoplexes and (C) LP-TC-1-12 lipoplexes were used. Each column represents the mean $+\mathrm{SD}(\mathrm{n}=3-5) .{ }^{*} \mathrm{P}<0.05,{ }^{* *} \mathrm{P}<0.01$ and ${ }^{* * * *} \mathrm{P}<0.001$ vs. non-PEGylated siRNA lipoplex. PEG, polyethylene glycol; siRNA, small interfering RNA; LP-DDAB, DDAB liposome; LP-DOTAP, DOTAP liposome; LP-TC-1-12, TC-1-12 liposome.

lipoplexes with PEG-DSG, PEG-Chol, and PEG-CS tended not to markedly inhibit the gene-silencing effects compared with PEGylation with PEG-DSPE (inhibitory effects in gene-silencing by PEGylation: PEG-DSPE > PEG-DSG $>$ PEG-Chol > PEG-CS), indicating that PEG-DSG, PEG-Chol, and PEG-CS may be more easily released from PEGylated siRNA lipoplexes, compared with PEG-DSPE. In particular, PEG-CS coating of siRNA lipoplexes did not inhibit gene-silencing effects by siRNA lipoplexes regardless of the cationic lipid type, suggesting that PEG coating via electrostatic interaction may be easily detached from siRNA lipoplexes compared with those by incorporation of PEG-lipid derivatives into the liposomal membrane via a lipid anchor. Regarding the PEG-DSPE, the electrostatic interaction between the phosphate group of PEG-DSPE and amine group of cationic lipids can remain stably on the surface of siRNA lipoplexes (10). From these results, the inhibition of the gene-silencing effects by PEGylation was largely affected by the anchor of PEG derivatives in PEGylated siRNA lipoplexes.

Cytotoxicity by PEGylated siRNA lipoplexes. To examine the effects of the anchor type of the PEG derivatives on cytotoxicity by PEGylated siRNA lipoplexes, we investigated cell viabilities at $24 \mathrm{~h}$ after transfection into MCF-7 cells with PEGylated siRNA lipoplexes. Non-PEGylated siRNA lipoplexes did not induce cytotoxicity regardless of the cationic lipid types in cationic liposomes ( $>90 \%$ in cell viability) (Fig. 4$)$. However, LP-DDAB-PEG-DMPE, LP-DDAB-PEG-DPPE, LP-DDAB-PEG-DPG, LP-DOTAP-PEG-Chol, and LP-DOTAP-PEG-CS lipoplexes increased cytotoxicity by PEGylation (60-80\% cell viability). In contrast, the other PEGylated lipoplexes did not show significant cytotoxicity.

Interaction with erythrocytes and PEGylated siRNA lipoplexes. To prevent aggregation of siRNA lipoplexes with blood components such as erythrocytes after systemic injection, the surface of siRNA lipoplexes has often been modified with PEG. To examine this effect, non-PEGylated and PEGylated siRNA lipoplexes were added into erythrocyte suspensions. Of the non-PEGylated cationic liposomes, all the siRNA lipoplexes induced agglutination after mixing with the erythrocyte suspension regardless of the cationic lipid type in the liposomal formulation (Fig. 5). However, in PEGylated siRNA lipoplexes, PEG on the surface of siRNA lipoplexes may prevent agglutination with erythrocytes with increasing amounts of PEG-lipid derivatives or PEG-CS in the liposomal formulation (Fig. 5). From this result, all the PEGylated siRNA lipoplexes were able to prevent aggregation with erythrocytes regardless of the anchor type of the PEG derivatives.

Biodistribution of siRNA after intravenous injection of PEGylated siRNA lipoplexes. To investigate the effects of the anchor of PEG derivatives in PEGylated siRNA 
A

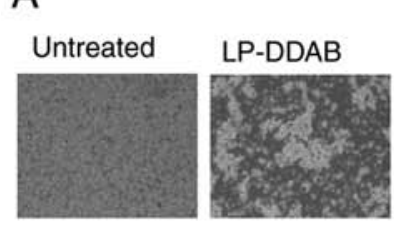

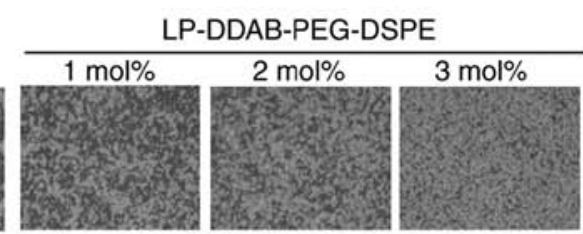

LP-DDAB-PEG-DPPE

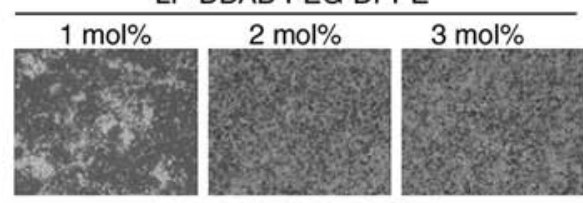

LP-DDAB-PEG-DMPE

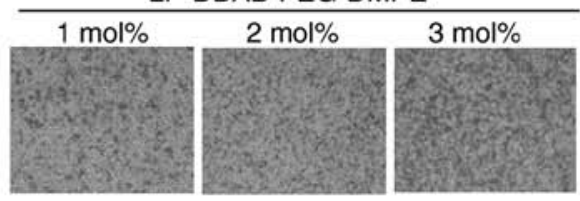

LP-DDAB-PEG-Chol

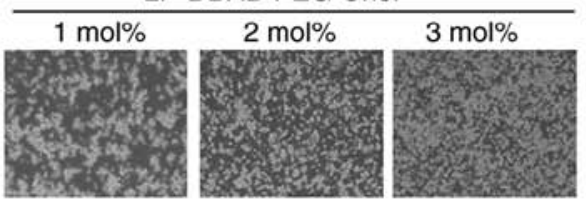

B

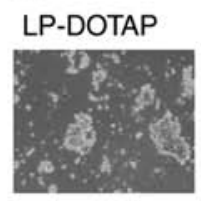

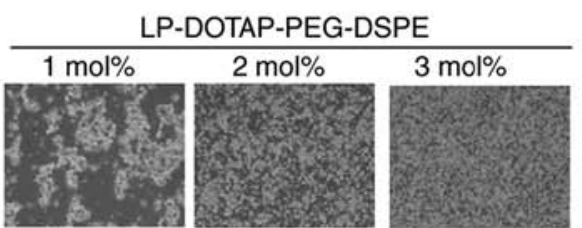

LP-DOTAP-PEG-DSG

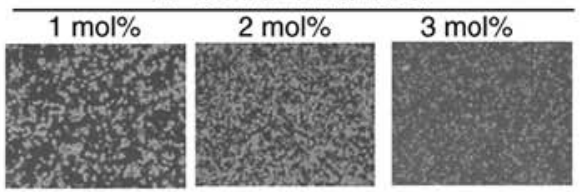

LP-DOTAP-PEG-Chol

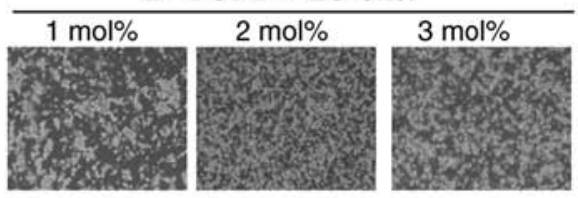

LP-DOTAP-PEG-CS

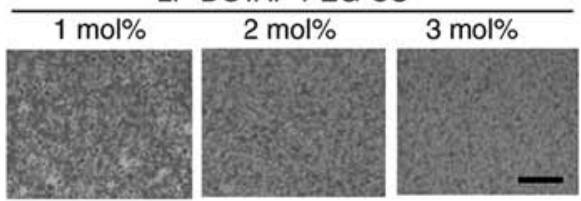

C
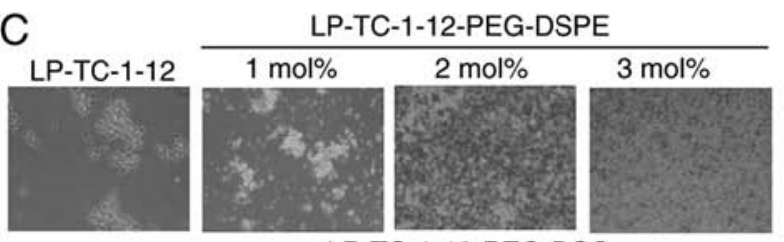

LP-TC-1-12-PEG-DSG

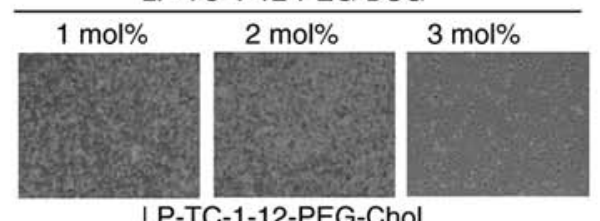

LP-TC-1-12-PEG-Chol

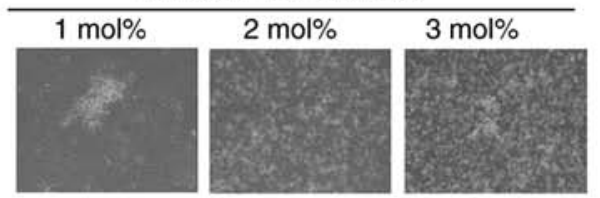

LP-TC-1-12-PEG-CS

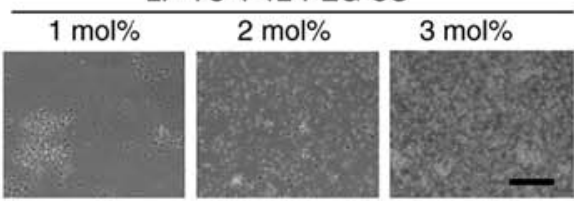

Figure 5. Effects of PEG-derivatives in cationic liposomes on the agglutination of PEGylated siRNA lipoplexes with erythrocytes. Non-PEGylated and 1,2 or 3 mol\% PEGylated siRNA lipoplexes containing $2 \mu \mathrm{g}$ siRNA were added to erythrocyte suspensions and agglutination was observed using phase contrast microscopy. (A) LP-DDAB lipoplexes, (B) LP-DOTAP lipoplexes and (C) LP-TC-1-12 lipoplexes were used (scale bar, $200 \mu$ m). PEG, polyethylene glycol; siRNA, small interfering RNA; LP-DDAB, DDAB liposome; LP-DOTAP, DOTAP liposome; LP-TC-1-12, TC-1-12 liposome.

lipoplexes on the biodistribution of siRNA after intravenous injection, we intravenously injected non-PEGylated and PEGylated lipoplexes with Cy5.5-siRNA into mice and observed the biodistribution of siRNA at $1 \mathrm{~h}$ after injection. In non-PEGylated siRNA lipoplexes, LP-DOTAP, LP-DDAB, and LP-TC-1-12 lipoplexes largely accumulated in the lungs (Figs. 6 and 7), indicating that positively charged siRNA lipoplexes bound to blood components such as erythrocytes in the blood circulation, and the agglutinates were entrapped in the highly extended lung capillaries. In contrast, in DDAB-based PEGylated siRNA lipoplexes, PEGylation of LP-DDAB lipoplexes with PEG-DSPE, PEG-DSG, PEG-Chol, or PEG-CS largely accumulated in the liver with increasing amounts of the derivatives (Figs. 6A and 7A), suggested that 

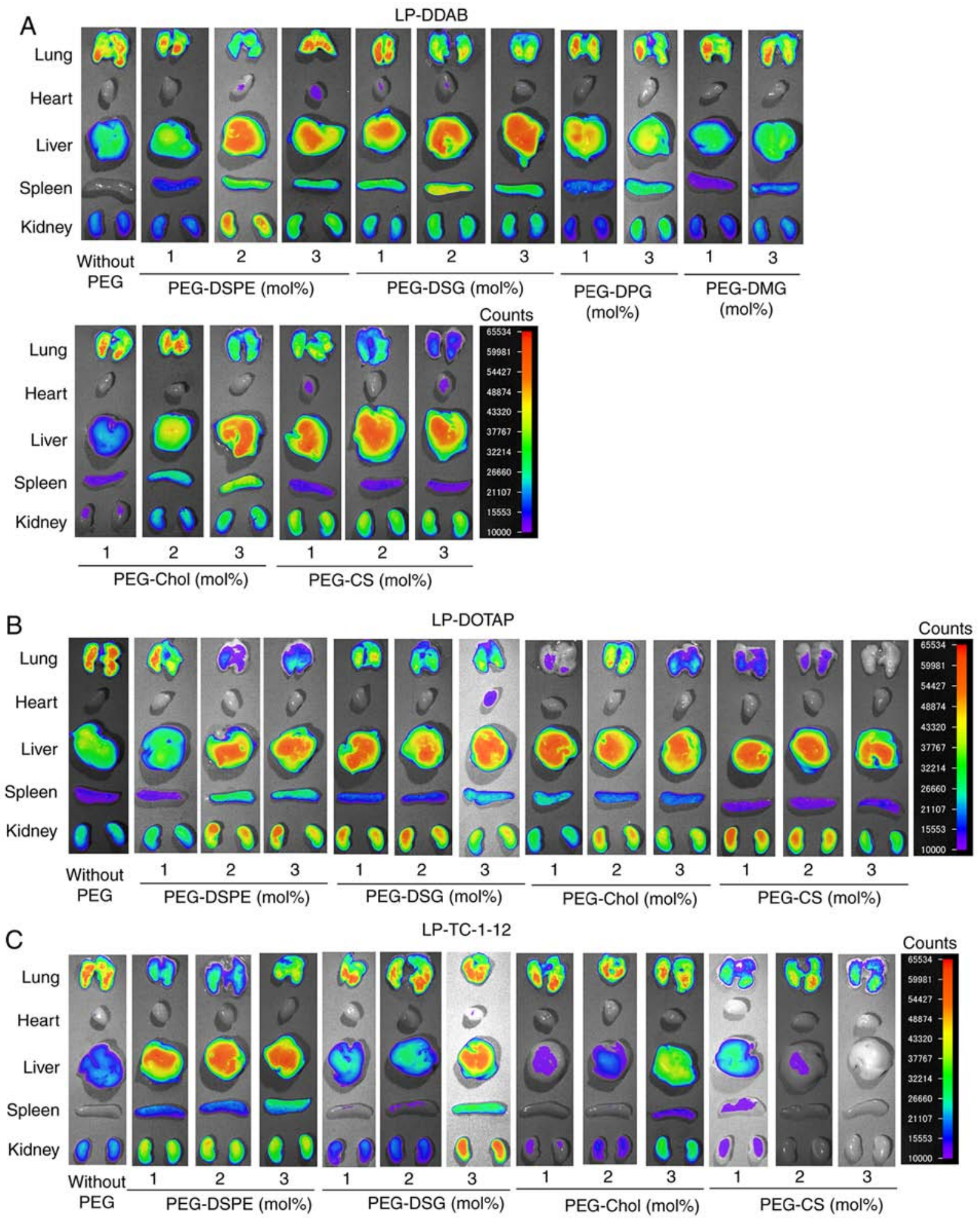

Counts

Figure 6. Effects of PEG-derivatives in cationic liposomes on the biodistribution of siRNA in mice at $1 \mathrm{~h}$ after intravenous injection with PEGylated siRNA lipoplexes. Non-PEGylated and PEGylated siRNA lipoplexes were prepared by mixing non-PEGylated and PEGylated cationic liposomes, respectively, with $20 \mu \mathrm{g}$ Cy5.5-siRNA, and were administered intravenously to mice. Ex vivo images of dissected tissues were obtained $1 \mathrm{~h}$ after intravenous injection. The exposure time for the detection of Cy5.5 fluorescence was $5 \mathrm{sec}$. Fluorescence intensity is illustrated using a color-coded scale (red is maximum, purple is minimum). (A) LP-DDAB lipoplexes, (B) LP-DOTAP lipoplexes and (C) LP-TC-1-12 lipoplexes were used. PEG, polyethylene glycol; siRNA, small interfering RNA; LP-DDAB, DDAB liposome; LP-DOTAP, DOTAP liposome; LP-TC-1-12, TC-1-12 liposome.

PEGylation can prevent agglutination in the blood circulation. However, PEGylation of LP-DDAB lipoplexes with PEG-DPG, or PEG-DMG did not largely affect the biodistribution of siRNA even at 3 mol\% PEGylation, compared with non-PEGylated LP-DDAB lipoplexes (Figs. 6A and 7A). These results indicated that PEG-DPG and PEG-DMG were rapidly released from PEGylated siRNA lipoplexes in the blood circulation, and did not prevent the interaction with blood components. In DOTAP-based PEGylated siRNA lipoplexes, PEGylation of LP-DOTAP lipoplexes with PEG-DSPE, 

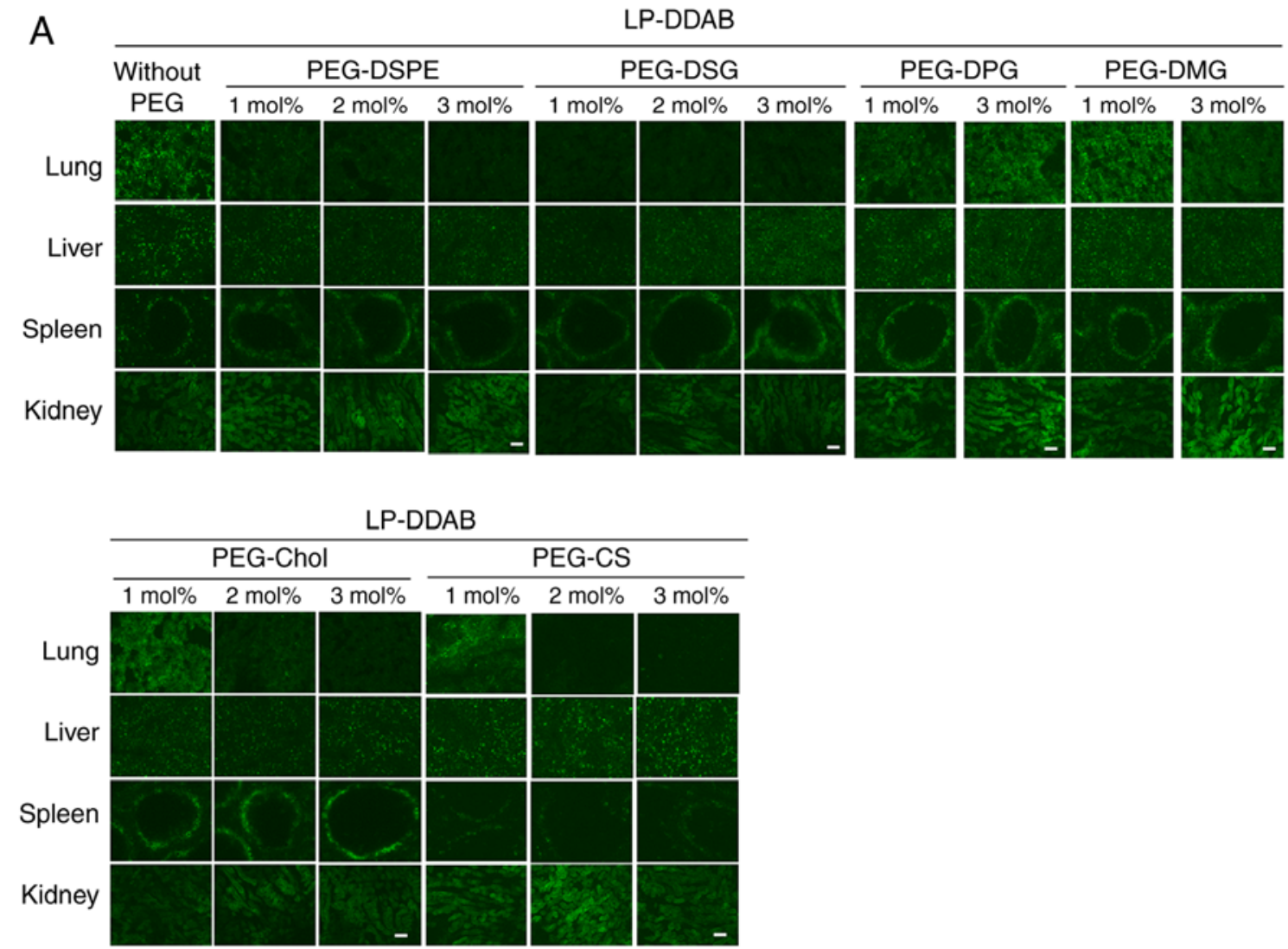

B

\begin{tabular}{|c|c|c|c|c|c|c|c|c|c|c|c|c|}
\hline \multirow{3}{*}{$\begin{array}{c}\text { Without } \\
\text { PEG }\end{array}$} & \multicolumn{12}{|c|}{ LP-DOTAP } \\
\hline & \multicolumn{3}{|c|}{ PEG-DSPE } & \multicolumn{3}{|c|}{ PEG-DSG } & \multicolumn{3}{|c|}{ PEG-Chol } & \multicolumn{3}{|c|}{ PEG-CS } \\
\hline & $1 \mathrm{~mol} \%$ & $2 \mathrm{~mol} \%$ & $3 \mathrm{~mol} \%$ & $1 \mathrm{~mol} \%$ & $2 \mathrm{~mol} \%$ & $3 \mathrm{~mol} \%$ & $1 \mathrm{~mol} \%$ & $2 \mathrm{~mol} \%$ & $3 \mathrm{~mol} \%$ & $1 \mathrm{~mol} \%$ & $2 \mathrm{~mol} \%$ & $3 \mathrm{~mol} \%$ \\
\hline & & - & & & & & & & & & & \\
\hline & & & & & & & & & & & & \\
\hline & & & & & & & & & & & & \\
\hline & & & & & & & & & & & 75e & 403 \\
\hline
\end{tabular}

C

LP-TC-1-12

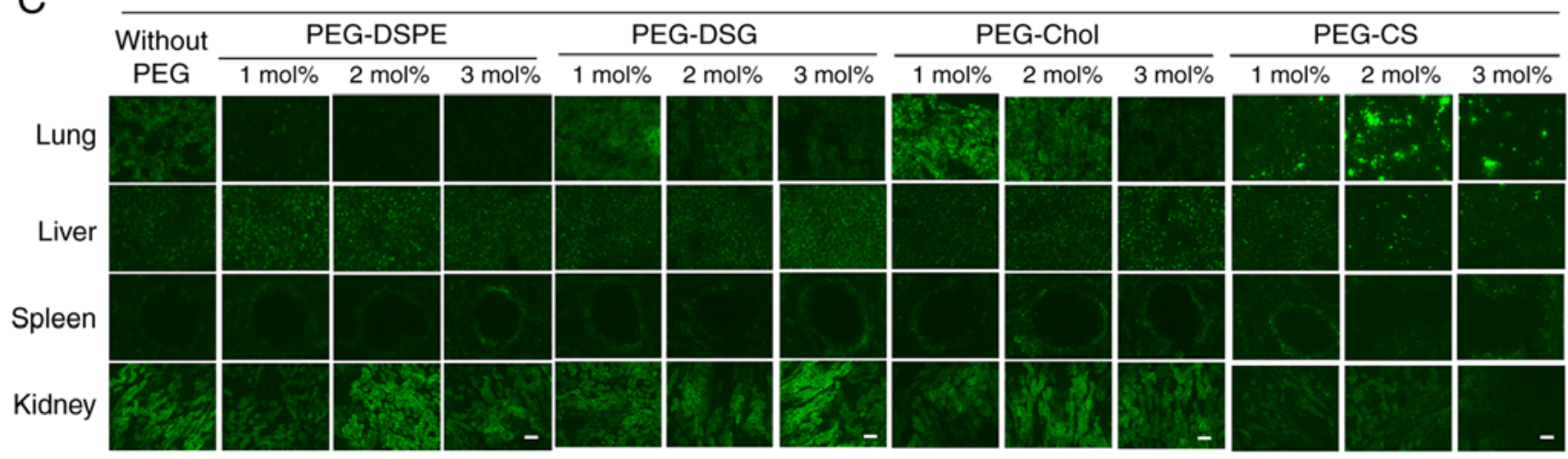

Figure 7. Effects of PEG-derivatives in cationic liposomes on the biodistribution of siRNA in mice at $1 \mathrm{~h}$ after intravenous injection of PEGylated siRNA lipoplexes. Non-PEGylated and PEGylated siRNA lipoplexes were prepared by mixing non-PEGylated and PEGylated cationic liposomes, respectively, with $20 \mu \mathrm{g}$ Cy5.5-siRNA, and were administered intravenously to mice. (A) LP-DDAB lipoplexes, (B) LP-DOTAP lipoplexes and (C) LP-TC-1-12 lipoplexes were used. The localization of Cy5.5-siRNA in tissues was examined $1 \mathrm{~h}$ after intravenous injection. Green signals indicated localization of Cy5.5-siRNA (scale bar, $100 \mu \mathrm{m}$ ). PEG, polyethylene glycol; siRNA, small interfering RNA; LP-DDAB, DDAB liposome; LP-DOTAP, DOTAP liposome; LP-TC-1-12, TC-1-12 liposome. 
PEG-DSG, PEG-Chol, or PEG-CS largely accumulated in the liver with increasing amounts of the PEG derivatives (Figs. 6B and 7B). Furthermore, in TC-1-12-based PEGylated siRNA lipoplexes, PEGylation of LP-TC-1-12 lipoplexes with PEG-DSPE largely accumulated in the liver even at $1 \mathrm{~mol} \%$ PEGylation, and PEGylation of LP-TC-1-12 lipoplexes with PEG-DSG or PEG-Chol reduced the accumulation of siRNA in the lungs with increasing amounts of the PEG derivatives (2-3 mol\% PEGylation) (Figs. 6C and 7C). These results suggested that PEGylation with PEG-DSG, PEG-Chol, and PEG-CS can prevent aggregation with blood components as well as that with PEG-DSPE regardless of the cationic lipid types in PEGylated siRNA lipoplexes.

In DDAB-based siRNA lipoplexes, injection of PEGylated siRNA lipoplexes with PEG lipid derivatives with dialkyl (C14-C16) chains induced siRNA accumulation in the lungs, whereas dialkyl (C18) chains induced siRNA accumulation in the liver (Figs. 6A and 7A). LP-DDAB-PEG-DSPE and LP-DDAB-PEG-DSG lipoplexes prevented agglutination with erythrocytes in the blood circulation and led to an increase in the accumulation of siRNA in the liver. In contrast, LP-DDAB-PEG-DPG and LP-DDAB-PEG-DMG lipoplexes accumulated in the lungs after intravenous injection, indicated that PEG-DPG and PEG-DMG may be quickly released from siRNA lipoplexes in blood circulation, resulting in agglutination with erythrocytes. These results indicated that siRNA biodistribution after intravenous injection of siRNA lipoplexes was strongly affected by the length of the PEG anchor in PEGylated siRNA lipoplexes. Moreover, regardless of the cationic lipid types in cationic liposomes, PEGylation with PEG-DSG, PEG-Chol, and PEG-CS can prevent aggregation with erythrocytes (Fig. 5) and decrease the accumulation in the lungs (Figs. 6 and 7) as well as that with PEG-DSPE although they did not markedly inhibit in vitro gene-silencing effects (Fig. 3). These results suggested that PEG-DSG, PEG-Chol, and PEG-CS in PEGylated siRNA lipoplexes may be gradually released from the lipoplexes; therefore, PEGylation with PEG-DSG, PEG-Chol, and PEG-CS may temporally stabilize siRNA lipoplexes in the blood circulation, resulting in a decrease in siRNA accumulation in the lungs. As a result, the PEGylated siRNA lipoplexes with PEG-DSG, PEG-Chol, and PEG-CS can accumulate in the liver without a loss of transfection activity by PEGylation; therefore, they may have potential as vectors for the delivery of siRNA therapeutics to the liver or liver metastasis. However, further studies should be performed to examine the effects of PEG anchors in PEGylated siRNA lipoplexes on hepatic toxicity and in vivo gene-silencing activity after intravenous injection.

Generally, stable PEGylation of siRNA lipoplexes strongly inhibits cellular uptake and endosomal escape, which result in a significant loss of RNAi activity. For successful siRNA delivery in vivo, a crucial problem associated with the use of PEG must be overcome. One way to solve this problem is to use cleavable PEG-lipid derivatives that release the PEG moiety by cleavage of a linker between PEG and the anchor lipid when exposed to the appropriate stimulus at the target site $(7,21)$. Most cleavable PEG derivatives have been designed to be cleaved in response to the extracellular or intracellular microenvironment such as temperature, $\mathrm{pH}$, specific enzymes, etc. However, the cleavage of a linker between PEG and the anchor lipid in response to a stimulus may be inefficient in some cases because PEG on the surface of siRNA lipoplexes will reduce association with stimuluses such as specific enzymes. Another strategy is to use releasable PEG derivatives that are released from siRNA lipoplexes as time advances although these can prevent non-specific association in blood circulation. The advantage of using releasable PEG derivatives is the ease and relatively low cost of preparation of PEGylated liposomes. Therefore, PEGylation of siRNA lipoplexes with releasable PEG-DSG, PEG-Chol, and PEG-CS may have the potential to improve systemic stability without a loss of transfection activity by PEGylation.

PEGylation of siRNA lipoplexes generally lowers the efficiency of siRNA-mediated gene-silencing in vitro and in vivo. In this study, we examined the effects of PEG anchors in PEGylated siRNA lipoplexes on in vitro gene-silencing effects and biodistribution after intravenous injection. PEGylation of siRNA lipoplexes with PEG-DSG, PEG-Chol, and PEG-CS tended not to greatly inhibit the gene-silencing effects compared with PEGylation with PEG-DSPE. However, PEG-DSG, PEG-Chol, and PEG-CS in PEGylated siRNA lipoplexes may prevent agglutination in the blood circulation and decrease the accumulation of siRNA in the lungs after systemic injection. From these findings, releasable PEGylation of siRNA lipoplexes with PEG-DSG, PEG-Chol, and PEG-CS may improve the systemic stability after intravenous injection without loss of transfection activity by PEGylation.

\section{Acknowledgements}

The authors would like to thank Ms. Naho Egashira, Ms. Asuka Sasaki, Ms. Yui Sugawara, Ms. Huka Nakajima, Ms.Azumi Otake, Mr. Ryota Shimizu,Ms. Yukiha,Mori and Ms. Haruka Takahashi (Department of Molecular Pharmaceutics, Hoshi University) for assistance with experimental work (in vitro gene-silencing effect).

\section{Funding}

The current project was supported in part by a Grant-in-Aid for Scientific Research (C) from the Japan Society for the Promotion of Science (grant no. 20K07142).

\section{Availability of data and materials}

The datasets used and/or analyzed during the current study are available from the corresponding author on reasonable request.

\section{Authors' contributions}

YH conceived and designed the study. Experiments were performed by KT, SS, KO and HO. YH wrote the manuscript. All authors read and approved the final manuscript.

\section{Ethics approval and consent to participate}

All animal experiments were conducted in accordance with the 'Guide for the Care and Use of Laboratory Animals' adopted by the Institutional Animal Care and Use Committee 
of Hoshi University (Tokyo, Japan; accredited by the Ministry of Education, Culture, Sports, Science and Technology). Ethical approval for the current study was obtained from the Institutional Animal Care and Use Committee of Hoshi University (approval no. 30-072).

\section{Patient consent for publication}

Not applicable.

\section{Competing interests}

The authors declare that they have no competing interests.

\section{References}

1. Wilson RC and Doudna JA: Molecular mechanisms of RNA interference. Annu Rev Biophys 42: 217-239, 2013.

2. Chen X, Mangala LS, Rodriguez-Aguayo C, Kong X, Lopez-Berestein G and Sood AK: RNA interference-based therapy and its delivery systems. Cancer Metastasis Rev 37: 107-124, 2018

3. Zhang S, Zhi D and Huang L: Lipid-based vectors for siRNA delivery. J Drug Target 20: 724-735, 2012.

4. Zatsepin TS, Kotelevtsev YV and Koteliansky V: Lipid nanoparticles for targeted siRNA delivery-going from bench to bedside. Int J Nanomedicine 11: 3077-3086, 2016.

5. Zhang Y, Satterlee A and Huang L: In vivo gene delivery by nonviral vectors: Overcoming hurdles? Mol Ther 20: 1298-1304, 2012.

6. Xia Y, Tian J and Chen X: Effect of surface properties on liposomal siRNA delivery. Biomaterials 79: 56-68, 2016.

7. Hatakeyama $\mathrm{H}$, Akita $\mathrm{H}$ and Harashima $\mathrm{H}$ : The polyethyleneglycol dilemma: Advantage and disadvantage of PEGylation of liposomes for systemic genes and nucleic acids delivery to tumors. Biol Pharm Bull 36: 892-899, 2013.

8. Hattori Y, Shimizu S, Ozaki KI and Onishi H: Effect of cationic lipid type in Folate-PEG-modified cationic liposomes on folate receptor-mediated siRNA transfection in tumor cells. Pharmaceutics 11: 181, 2019.

9. Sonoke S, Ueda T, Fujiwara K, Sato Y, Takagaki K, Hirabayashi K, Ohgi $\mathrm{T}$ and Yano J: Tumor regression in mice by delivery of Bcl-2 small interfering RNA with pegylated cationic liposomes. Cancer Res 68: 8843-8851, 2008.

10. Song F, Sakurai N, Okamoto A, Koide H, Oku N, Dewa T and Asai T: Design of a novel PEGylated liposomal vector for systemic delivery of siRNA to solid tumors. Biol Pharm Bull 42: 996-1003, 2019.
11. Chen S, Tam YY, Lin PJ, Leung AK, Tam YK and Cullis PR Development of lipid nanoparticle formulations of siRNA for hepatocyte gene silencing following subcutaneous administration. J Control Release 196: 106-112, 2014.

12. Nakamura T, Noma Y, Sakurai Y and Harashima H: Modifying cationic liposomes with cholesteryl-PEG prevents their aggregation in human urine and enhances cellular uptake by bladder cancer cells. Biol Pharm Bull 40: 234-237, 2017.

13. Hattori Y, Nakamura T, Ohno H, Fujii N and Maitani Y: siRNA delivery into tumor cells by lipid-based nanoparticles composed of hydroxyethylated cholesteryl triamine. Int J Pharm 443: 221-229, 2013.

14. Hattori Y, Hara E, Shingu Y, Minamiguchi D, Nakamura A, Arai S, Ohno H, Kawano K, Fujii N and Yonemochi E: siRNA delivery into tumor cells by cationic cholesterol derivative-based nanoparticles and liposomes. Biol Pharm Bull 38: 30-38, 2015.

15. Hattori Y, Arai S, Kikuchi T, Ozaki K, Kawano K and Yonemochi E: Therapeutic effect for liver-metastasized tumor by sequential intravenous injection of anionic polymer and cationic lipoplex of siRNA. J Drug Target 24: 309-317, 2016.

16. Dawlee S, Sugandhi A, Balakrishnan B, Labarre D and Jayakrishnan A: Oxidized chondroitin sulfate-cross-linked gelatin matrixes: A new class of hydrogels. Biomacromolecules 6 : 2040-2048, 2005.

17. Sasatsu M, Onishi $\mathrm{H}$ and Machida Y: Preparation of a PLA-PEG block copolymer using a PLA derivative with a formyl terminal group and its application to nanoparticulate formulation. Int J Pharm 294: 233-245, 2005.

18. Hattori Y, Nakamura A, Hanaya S, Miyanabe Y, Yoshiike Y, Kikuchi T, Ozaki KI and Onishi H: Effect of chondroitin sulfate on siRNA biodistribution and gene silencing effect in mice after injection of siRNA lipoplexes. J Drug Deliv Sci Tech 41: 401-409, 2017.

19. Hattori Y, Nakamura A, Arai S, Nishigaki M, Ohkura H, Kawano K, Maitani Y and Yonemochi E: In vivo siRNA delivery system for targeting to the liver by poly-l-glutamic acid-coated lipoplex. Results Pharma Sci 4: 1-7, 2014.

20. Hattori Y, Yamasaku H and Maitani Y: Anionic polymer-coated lipoplex for safe gene delivery into tumor by systemic injection. J Drug Target 21: 639-647, 2013

21. Fang Y, Xue J, Gao S, Lu A, Yang D, Jiang H, He Y and Shi K: Cleavable PEGylation: A strategy for overcoming the 'PEG dilemma' in efficient drug delivery. Drug Deliv 24 (sup1): 22-32, 2017.

This work is licensed under a Creative Commons Attribution-NonCommercial-NoDerivatives 4.0 International (CC BY-NC-ND 4.0) License. 